\title{
Analysis and Study of Flow Patterns and Sediment Deposition in the Forebay of a Forward Intake Pumping Station
}

\section{Haidong WANG}

Ningxia University

Chunguang LI ( $\nabla$ cglizd@hotmail.com )

North Minzu University

\section{Suiju LV}

North Minzu University

Lulu SONG

Ningxia University

\section{Research Article}

Keywords: pump station, sediment deposition, vortices, numerical simulation

Posted Date: January 7th, 2022

DOI: https://doi.org/10.21203/rs.3.rs-1226763/v1

License: (c) (i) This work is licensed under a Creative Commons Attribution 4.0 International License. Read Full License 


\title{
Analysis and study of flow patterns and sediment deposition
}

\section{in the forebay of a forward intake pumping station}

\author{
Haidong Wang ${ }^{1}$, Chunguang $\mathrm{Li}^{2}$, Suiju Lv ${ }^{3}$, Lulu Song ${ }^{1}$ \\ 1. College of Civil and Hydraulic Engineering, Ningxia University, Yinchuan 750021, Ningxia, China \\ 2. College of Mathematics and Information Science, North Minzu University, Yinchuan 750021, China \\ 3. College of Civil Engineering, North Minzu University, Yinchuan 750021, Ningxia, China
}

\begin{abstract}
In Northwest China, the sediment concentration of the Yellow River is high. A project to investigate the operation of a pumping station shows that the flow patterns in the forebay and inlet tank are disordered, and there is sediment deposition that endangers the normal operation and safety of the pumping station. To solve this problem, the three-dimensional two-phase water-sediment flow in the forebay of the pumping station is modeled by using fluid simulation software, and diagrams of the sediment volume fraction content and vector distribution in the flow layers of different sections are obtained. Combined with the multiphase flow theory of mixtures and the realizable $k-\varepsilon$ turbulent kinetic energy equation, the location and formation mechanism of each vortex, as well as the area and degree of sediment deposition in the forebay, are analyzed. The actual engineering and numerical simulation results are compared to verify the accuracy of the simulation. The results show that the main reason for sediment deposition is the high sediment concentration of the Yellow River, but the flow pattern disorder is affected by a specific design defect of the forebay, which makes the sediment deposition worse. The results of this study provide specific guidance and methods for the construction and transformation of the forebay of the pump station in the future; construction to weaken the return area to a certain extent can reduce the degree of sedimentation.
\end{abstract}

Key words: pump station; sediment deposition; vortices; numerical simulation

\section{Introduction}

China is a largely agricultural country that is based on agricultural development, and the foundation of agricultural development is land and water. Pumping stations play an indispensable role in solving irrigation problems. According to national statistics, the annual average drainage volume of large-scale pumping stations is as high as 40 billion $\mathrm{m}^{3}$, which provides protection for domestic water supplies in many cities and rural irrigation water in China. The forebay, which is an important part of the pumping station, can smoothly spread water from the diversion canal to the inlet tank. Since the pumping station depends mainly on the inlet tank for extraction of water, the flow pattern in the forebay directly affects the normal operation and drainage efficiency of the station. However, for pumping stations in Northwest China, little is known about the flow pattern in the forebay. The main reason is that the Yellow River is the main irrigation river in the arid area of Northwest China, but it is also the river with the largest sediment concentration in the world ${ }^{1}$, and its annual average sediment concentration is as high as $30 \sim 150 \mathrm{~kg} / \mathrm{m}^{3}$. Therefore, a large amount of sediment deposition easily forms in the forebay of pump stations in this area, which has a great impact on the water supply system of the pump station. 
With the rapid development of computer technology and the continuous improvement in computational fluid dynamics ${ }^{2}$, the two technologies are combined to carry out numerical simulations for the forebay, inlet tank and culvert of the pump station. In terms of numerical simulation research, remarkable achievements have been made in recent years: Constantinescu and Patel $^{3}$ used numerical simulations of turbulence in a rectangular tank with a Reynolds number of 60,000 to predict the position and intensity of the free surface and Coanda vortex. Hwang et $\mathrm{al}^{4}$. using numerical simulations and corresponding tests, obtained the velocity field and density field through the direct simulation Monte Carlo (DSMC) technique. Adrian and Ronald ${ }^{5}$ analyzed and studied the formation mechanism of a hairpin vortex in wall turbulence through a numerical simulation. At the same time, a gradually increasing number of researchers are applying numerical simulations of the forebays of pump stations. $\mathrm{Xi}$ and $\mathrm{Lu}^{6}$ analyzed the formation mechanism of nonsymmetric adhesive vortices in lateral pumping stations by using numerical simulation combined with Reynolds shear stress distributions. Jie-min et $\mathrm{al}^{7}$. used the renormalization group (RNG) $k-\varepsilon$ model and volume of fluid (VOF) method to analyze the vortex position and structure shape. Xue-lin et $\mathrm{al}^{8}$. applied the coupled lattice Boltzmann method-sub-grid stress (LBM-SGS) model to the analysis of the forebay of a pump station and predicted the secondary flow and water level flow characteristics of the forebay.

However, numerical simulations are rarely applied to the analysis of two-phase water-sediment flows in the forebays of pumping stations. When the sediment pattern is too high, it aggravates flow pattern disorder in the forebay of a pump station, and the bad flow pattern leads to large amounts of sediment deposition in the forebay. To solve the problem of sedimentation, the complex flow pattern of two-phase water-sand flow must be considered fundamentally. Therefore, through an in-depth study of this kind of flow pattern, combined with computational fluid dynamics and numerical simulation technology, this paper studies and analyzes a particular two-phase water sediment flow, compares the results of the simulation with those of the actual engineering project, determines the design problems of the forebay, and provides reliable guidance and a basis for the new construction and modification of the forebay of the pump station in the future.

\section{Mathematical model}

2.1 Governing equations of the mixture model for multiphase flow

The Qingtongxia station is located on the mainstream of the Yellow River in the northwest of the second pumping station area. The measured average sediment concentration of the Yellow River Basin in the last five years is $1.45 \mathrm{~kg} / \mathrm{m}^{3}$. Therefore, various complex factors, such as sediment deposition, settlement and wide distribution range, must be considered for the flow pattern in the forebay of the second pump station. In this study, the mixture model in Fluent was selected for the calculations, mainly for the following reasons:

(1) The forebay flow of the second pump station is a complex two-phase flow of water and sediment with three-dimensional incompressible viscous turbulence, and heat transfer and transformation are ignored in this calculation;

(2) The mixture model can be simulated by solving the equations for momentum continuity of the mixture, the energy equation, the second phase volume fraction equation, and the algebraic 
expression of the relative velocity, and it has good consistency for modelling precipitation, eddies and low-load particles;

(3) When the sediment particles are widely distributed or the water sediment law is complex, the number of variables is less when using a mixture.

In summary, the mixture model is more suitable than other models for the calculation of water-sand flow.

The various equations are as follows (Ansys, 2017):

Continuity equation:

$$
\frac{\partial}{\partial t}\left(\rho_{m}\right)+\nabla \cdot\left(\rho_{m} \overrightarrow{v_{m}}\right)=0
$$

where $\rho_{m}$ is the mixing density and $\overrightarrow{v_{m}}$ is the mass average velocity.

$\rho_{m}$ is expressed as:

$$
\rho_{m}=\sum_{k=1}^{n} \alpha_{k} \rho_{k}
$$

$\overrightarrow{v_{m}}$ is expressed as follows:

$$
\overrightarrow{v_{m}}=\frac{\sum_{k=1}^{n} \alpha_{k} \rho_{k} \overrightarrow{v_{k}}}{\rho_{m}}
$$

where $\alpha_{k}$ is the volume fraction of phase $\mathrm{k}, \rho_{k}$ is the density of phase $\mathrm{k}$, and $\overrightarrow{v_{k}}$ is the velocity of phase $\mathrm{k}$.

The momentum equation of the mixture is obtained by summing the single momentum equations of all phases:

$$
\begin{aligned}
& \frac{\partial}{\partial t}\left(\rho_{m} \overrightarrow{v_{m}}\right)+\nabla \cdot\left(\rho_{m} \overrightarrow{v_{m} v_{m}}\right)=-\nabla p+\nabla \cdot\left[\mu_{m}\left(\nabla \overrightarrow{v_{m}}+\nabla{\overrightarrow{v_{m}}}^{T}\right)\right]+\rho_{m} \vec{g}+\vec{F} \\
&-\nabla \cdot\left(\sum_{k=1}^{n} \alpha_{k} \rho_{k} \overrightarrow{v_{d r, k}} \overrightarrow{v_{d r, k}}\right)
\end{aligned}
$$

where $\mathrm{n}$ is the number of phases, $\vec{F}$ is the volume force, $\mu_{m}$ is the mixed viscosity, and $\overrightarrow{v_{d r, k}}$ is the drift velocity of the second phase $\mathrm{K}$. In this study, it is the sediment particle phase.

$\mu_{m}$ is expressed as:

$$
u_{m}=\sum_{k=1}^{n} \alpha_{k} u_{k}
$$

$\mu_{k}$ is the viscosity of phase $\mathrm{K}$.

$\overrightarrow{v_{d r, k}}$ is expressed as:

$$
\overrightarrow{v_{d r, k}}=\overrightarrow{v_{k}}-\overrightarrow{v_{m}}
$$

The relative velocity $\overrightarrow{v_{p q}}$ is the velocity of the secondary phase (p sediment) relative to the main phase (q water);

$$
\overrightarrow{v_{p q}}=\overrightarrow{v_{p}}-\overrightarrow{v_{q}}
$$

The mass fraction of each phase $\mathrm{K}$ in the two phases of water and sediment is defined as:

$$
C_{k}=\frac{\alpha_{k} \rho_{k}}{\rho_{m}}
$$

The drift velocity and relative velocity $\overrightarrow{v_{p q}}$ are expressed by the following relationship:

$$
\overrightarrow{v_{d r, p}}=\overrightarrow{v_{p q}}-\sum_{k=1}^{n} \frac{\alpha_{k} \rho_{k}}{\rho_{m}} \overrightarrow{v_{k q}}
$$

Manninen et $\mathrm{al}^{9}$. assumed equilibrium in local space and derived the dispersion phase velocity from the algebraic equilibrium equation.

where the relative velocity is:

$$
\overrightarrow{v_{p q}}=\frac{\tau_{p}}{f_{\text {drag }}}-\frac{\left(\rho_{p}-\rho_{m}\right)}{\rho_{p}} \vec{\alpha}
$$


and $\tau_{p}$ is the particle relaxation time, $\vec{\alpha}$ is the acceleration of the secondary phase ( $\mathrm{p}$ sediment), $\rho_{p}$ is the density of the secondary phase ( $\mathrm{p}$ sediment), and $f_{\text {drag }}$ is the $\operatorname{drag}^{10}$ coefficient.

The particle relaxation time $\tau_{p}$ is:

$$
\tau_{p}=\frac{\rho_{p} d_{p}^{2}}{18 \mu_{q}}
$$

where $\mathrm{d}$ is the diameter of secondary phase (p sediment).

In the mixture model, according to the drift flux model, the acceleration of particles is replaced by gravity.

Therefore, the acceleration of the secondary phase (p sediment) $\vec{\alpha}$ is:

$$
\vec{\alpha}=\vec{g}-\left(\overrightarrow{v_{m}} \cdot \nabla\right) \overrightarrow{v_{m}}-\frac{\partial \overrightarrow{v_{m}}}{\partial t}
$$

$f_{\text {drag }}$ is:

$$
f_{\text {drag }}= \begin{cases}1+0.15 R e^{0.687} & R e \leq 1000 \\ 0.183 R e & R e \geq 1000\end{cases}
$$

In the mixture turbulence model, due to the dispersion of the momentum equation of the sediment particles, a diffusion term is added to the relative velocity. Therefore, the relative velocity equation is modified to:

$$
\overrightarrow{v_{p q}}=\frac{\left(\rho_{p}-\rho_{m}\right) d_{p}^{2}}{18 \mu_{q} f_{d r a g}} \vec{a}-\frac{\eta_{t}}{\sigma_{t}}\left(\frac{\nabla \alpha_{p}}{\alpha_{p}}-\frac{\nabla \alpha_{q}}{\alpha_{q}}\right)
$$

where $\sigma_{t}$ is the Prandt $/ / \mathrm{Schmidt}$ number ${ }^{11}$, which is set to 0.75 for this simulation, and $\eta_{t}$ is the turbulent diffusion coefficient, which is calculated from the continuous and dispersed pulsating velocity correlation equation:

$$
\begin{gathered}
\eta_{t}=C_{\mu} \frac{k^{3}}{\epsilon}\left(\frac{\gamma_{\gamma}}{1+\gamma_{\gamma}}\right)\left(1+C_{\beta} \zeta_{\gamma}^{2}\right)^{-1 / 2} \\
\zeta_{\gamma}=\frac{\left|\overrightarrow{v_{p q}}\right|}{\sqrt{\frac{2}{3} k}}
\end{gathered}
$$

where $C_{\beta}=1.8-1.35 \cos ^{2} \theta, \cos =\frac{\overrightarrow{v_{p q}} \cdot \overrightarrow{v_{p}}}{\left|\overrightarrow{v_{p q}}\right|\left|\overrightarrow{v_{p}}\right|}$ and $\gamma_{\gamma}$ are the time ratios between the time scale of the high-energy turbulent vortex and the particle relaxation time.

The volume fraction of the second phase sediment is:

$$
\frac{\partial}{\partial_{t}}\left(\alpha_{p} \rho_{p}\right)+\nabla \cdot\left(\alpha_{p} \rho_{p} \overrightarrow{v_{m}}\right)=-\nabla\left(\alpha_{p} \rho_{p} \overrightarrow{v_{d r, p}}\right)+\sum_{q=1}^{n}\left(\dot{m}_{q p}-\dot{m}_{p q}\right)
$$

\subsection{Turbulence model}

According to different methods of modeling the Reynolds stress term ${ }^{12}$, there are two types of Reynolds-averaged models : the eddy viscosity model and the Reynolds stress model. However, for this study, the Reynolds stress model is not suitable for the analysis of the flow pattern in the forebay of the pumping station because of it includes a large number of calculations.

The eddy viscosity model includes mainly the $k-\varepsilon$ model, $k-\omega$ model, and Spalart Allmaras model ${ }^{13,14}$. At present, the $k-\varepsilon$ model and $k-\omega$ model are the most widely used in simulations of pumping stations because both types of models are composed of two equations, and the calculation accuracy is high. For the numerical simulation of the diversion channel, forebay, and inlet tank of the pumping station, the $k-\varepsilon$ model is often used, in which the 
turbulent viscosity is expressed by the transport equation of turbulent kinetic energy $k$ and the turbulent dissipation rate $\varepsilon$. The $k-\varepsilon$ turbulence model can be divided into the standard $k-\varepsilon$ model, RNG $k-\varepsilon$ model, and realizable $k-\varepsilon$ model. The standard $k-\varepsilon$ model was proposed by Launder and Spaldingin $1974^{15}$. The model has good stability and high calculation accuracy. It is the most widely used and typical calculation model used in scientific research and practical engineering. The model obtains the values of $k$ and $\varepsilon$ by solving the equation of turbulent kinetic energy $k$ and the equation of dissipation rate $\varepsilon$ and then calculates the turbulent viscosity. The RNG $k-\varepsilon$ model is an improvement in the standard k- $\varepsilon$ model. It was derived based on the renormalization group method in $19866^{16}$ and improved in 1992 . After correcting the turbulent viscosity, the rotation and swirling conditions of the water flow are fully introduced in the average flow, and the spatial coordinate function is also included. The realizable $k-\varepsilon$ model adds the related content of rotation and curvature to the turbulent viscosity, keeps the Reynolds stress consistent with the real turbulence and realizes the Reynolds stress condition constraint ${ }^{17}$. The dissipation rate equation is modified according to the mean square eddy current pulsation dynamic equation, and the realizable $k-\varepsilon$ model is more effective than the standard $k-\varepsilon$ model and RNG $k-\varepsilon$ model in the numerical simulation of separate flows or secondary flows with complex flows. However, when it is easy to produce nonphysical turbulent viscosity problems, such as multireference systems and sliding grids, this kind of equation should be carefully selected. The equation of realizable $k-\varepsilon$ model is as follows:

The equation of turbulent kinetic energy $k$ is:

$$
\frac{\partial(\rho k)}{\partial t}+\frac{\partial\left(\rho k \mu_{i}\right)}{\partial x_{i}}=\frac{\partial}{\partial x_{j}}\left[\left(\mu+\frac{\mu_{t}}{\sigma_{k}}\right) \frac{\partial k}{\partial x_{j}}\right]+G_{k}-\rho \varepsilon
$$

The equation of turbulent energy dissipation rate $\varepsilon$ is:

$$
\frac{\partial(\rho \varepsilon)}{\partial t}+\frac{\partial\left(\rho \varepsilon \mu_{i}\right)}{\partial x_{i}}=\frac{\partial}{\partial x_{j}}\left[\left(\mu+\frac{\mu_{t}}{\sigma_{\varepsilon}}\right) \frac{\partial \varepsilon}{\partial x_{j}}\right]+\rho C_{1} E \varepsilon-\rho C_{2} \frac{\varepsilon^{2}}{k+\sqrt{v \varepsilon}}
$$

where $\sigma_{k}, \sigma_{\varepsilon}$, and $C_{2}$ are $1.0,1.2$, and 1.9 , respectively. $C_{1}=\max \left(0.43, \frac{\eta}{\eta+5}\right), \eta=$ $\left(2 E_{i j} \cdot E_{i j}\right)^{\frac{1}{2}} \frac{k}{\varepsilon}, E_{i j}=\frac{1}{2}\left(\frac{\partial u_{i}}{\partial x_{j}}+\frac{\partial u_{j}}{\partial x_{i}}\right), \mu_{t}=\rho C_{\mu} \frac{k^{2}}{\varepsilon}, C_{\mu}=\frac{1}{A_{0}+A_{S} U^{*} k / \varepsilon}, A_{0}=4.0, A_{S}=\sqrt{6} \cos \emptyset$, $\varnothing=\frac{1}{3} \cos ^{-1}(\sqrt{6} W), \quad W=\frac{E_{i j} E_{j k} E_{k j}}{\left(E_{i j} E_{i j}\right)^{\frac{1}{2}}}, \quad U^{*}=\sqrt{E_{i j} E_{i j}+\widetilde{\Omega}_{i j} \widetilde{\Omega}_{i j}}, \quad \widetilde{\Omega}_{i j}=\Omega_{i j}-2 \varepsilon_{i j k} \omega_{k}, \quad$ and $\Omega_{i j}=\bar{\Omega}-\varepsilon_{i j k} \omega_{k} \cdot \bar{\Omega}_{i j}$ is the time-averaged rotation rate tensor, which is obtained from the reference frame of the angular velocity $\omega_{k}$. For a flow field without rotation, $\widetilde{\Omega}_{i j} \widetilde{\Omega}_{i j}$ in $U^{*}$ in the above formula is 0 .

\subsection{Model establishment}

Based on the mixture model theory, the two-phase water sediment flow is established to carry out 1:1 three-dimensional reduction modeling and numerical simulation of the second pump station (Wulipo pump station) of the Yanhuanding Yellow River lifting project. The design drawing is shown in Figure 1. The model is mainly composed of a solid pump pool and fluid area, as shown in Figure 2. To consider the causes of sediment deposition, the average measured water inflow of the Qingtongxia station in the last 5 years (from June to September when the sediment content is highest) is 9.644 billion $\mathrm{m}^{3}$, and the average sediment inflow is 24 million tons. The average sediment concentration is converted to $2.49 \mathrm{~kg} / \mathrm{m}^{3}$ according to the sediment density 
$\rho=25,00 \mathrm{~kg} / \mathrm{m}^{3}$, and the calculated sediment volume percentage is $0.1 \%$. Therefore, ignoring the force of sediment on the flow and the interaction between sediment particles, the flow can be set as a steady flow and ignoring the influence of time change on the velocity. The steady flow satisfies the mathematical formula:

$$
\frac{\partial}{\partial t}=0
$$

Therefore, formulas (1), (4), and (17) can all be simplified as:

Continuity equation:

$$
\nabla \cdot\left(\rho_{m} \overrightarrow{v_{m}}\right)=0
$$

Momentum equation:

$$
\begin{gathered}
\nabla \cdot\left(\rho_{m} \overrightarrow{v_{m} v_{m}}\right)=-\nabla p+\nabla \cdot\left[\mu_{m}\left(\nabla \overrightarrow{v_{m}}+\nabla{\overrightarrow{v_{m}}}^{T}\right)\right]+\rho_{m} \vec{g}+\vec{F} \\
-\nabla \cdot\left(\sum_{k=1}^{n} \alpha_{k} \rho_{k} \overrightarrow{v_{d r, k}} \overrightarrow{v_{d r, k}}\right)
\end{gathered}
$$

The volume fraction of the second phase sediment is:

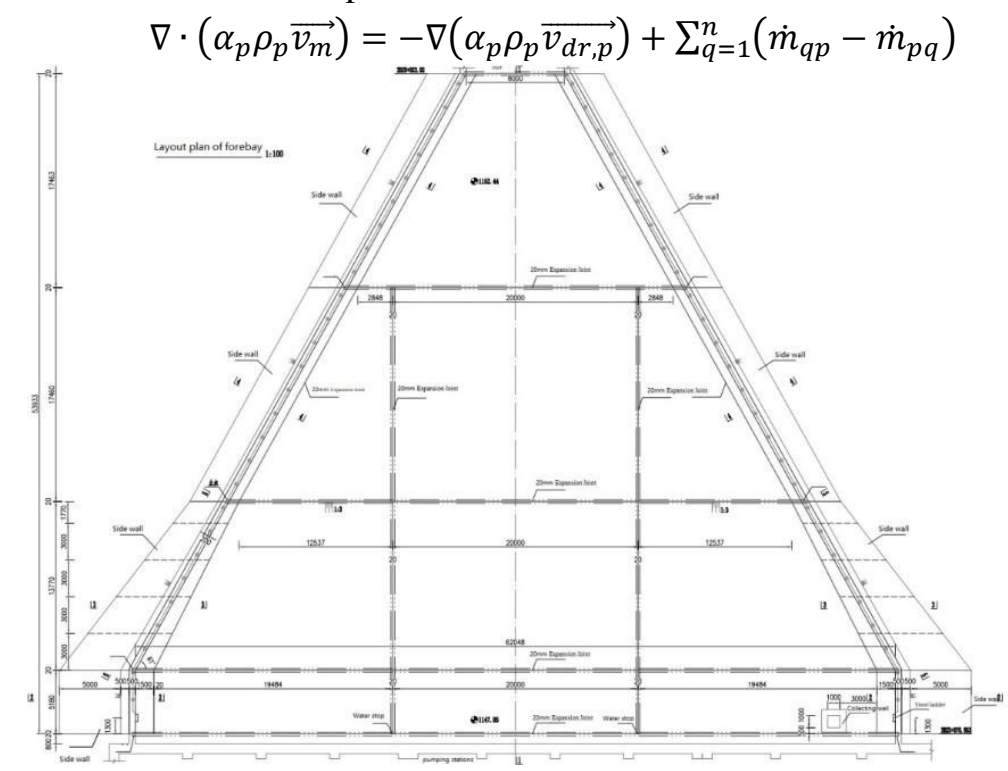

a. Structural layout of the forebay of the pump station

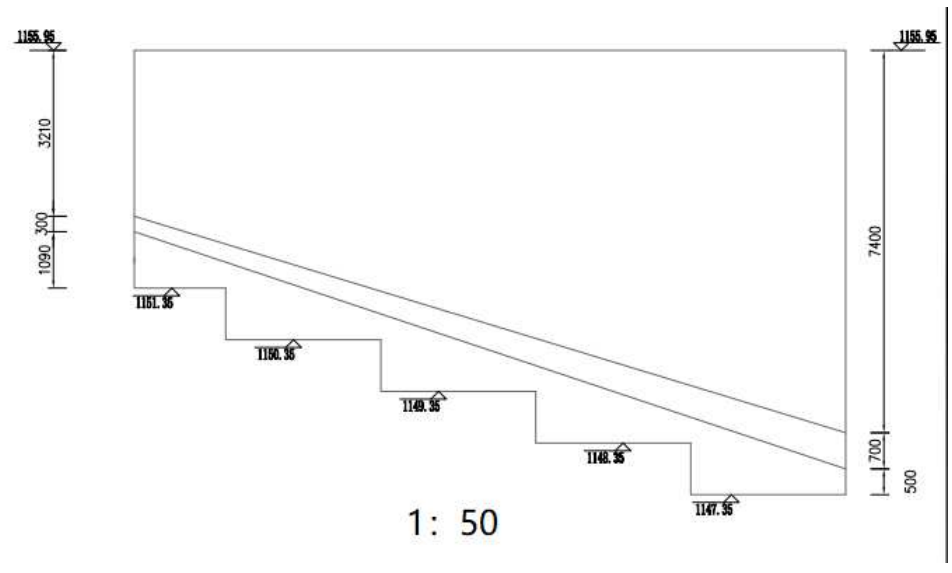

b. Wall thickness of the forebay of the pump station 


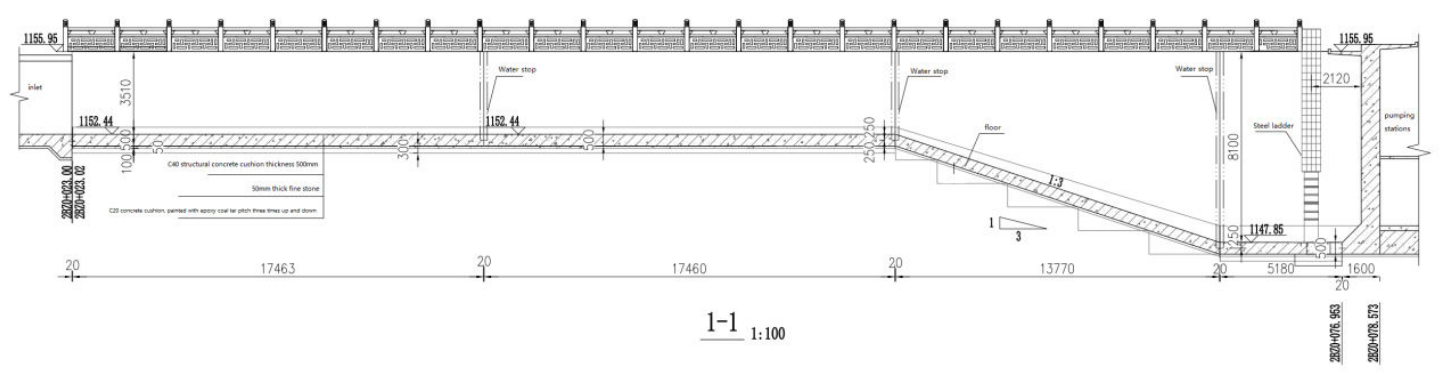

c. Longitudinal view of the forebay of the pump station

Figure 1 Design drawing of forebay of pump station

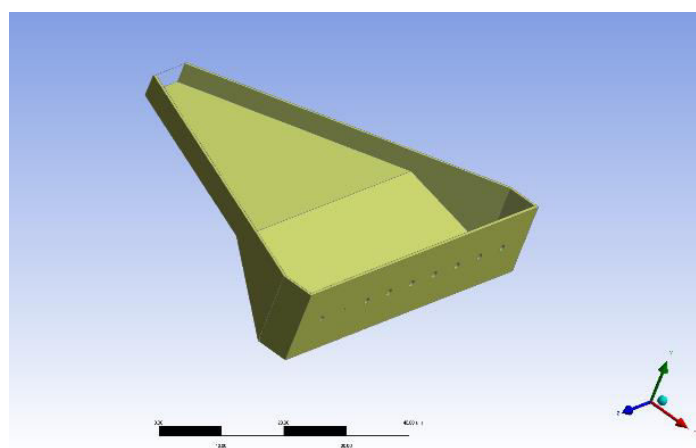

a. Three-dimensional diagram of the solid area in the forebay of the pump station

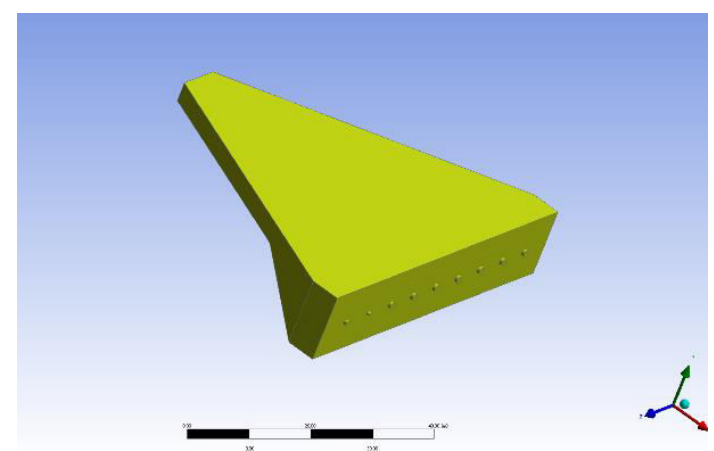

b. Three-dimensional diagram of the fluid area in the forebay of the pump station

Figure 2 Three-dimensional diagram of the forebay of the pump station

At the same time, the solid part in the pump station pool is suppressed in the calculation process, and only the two-phase water-sand flow in the fluid area is calculated to reduce the calculation amount and speed up the convergence of the calculation.

Due to the complex structure of the fluid region in two-phase water-sediment flow, an unstructured grid is used to divide the grid. To improve the calculation accuracy, the average grid size is set to $0.5 \mathrm{~m}$, and the inlet and outlet are partially dense. Hydraulic loss is adopted as the qualified parameter for measuring the grid number. When the overall grid number is 894,794 units, the hydraulic loss basically remains unchanged, in which the maximum volume is $3.89 e^{-2} \mathrm{~m}^{3}$, the minimum volume is $3.20 e^{-3} \mathrm{~m}^{3}$, the total volume of the water and sediment fluid domains is $1.32 e^{4} \mathrm{~m}^{3}$, the maximum area domain is $2.90 e^{-1} \mathrm{~m}^{2}$, and the minimum area domain is $3.19 e^{-2} m^{2}$, as shown in Figure 3 . 


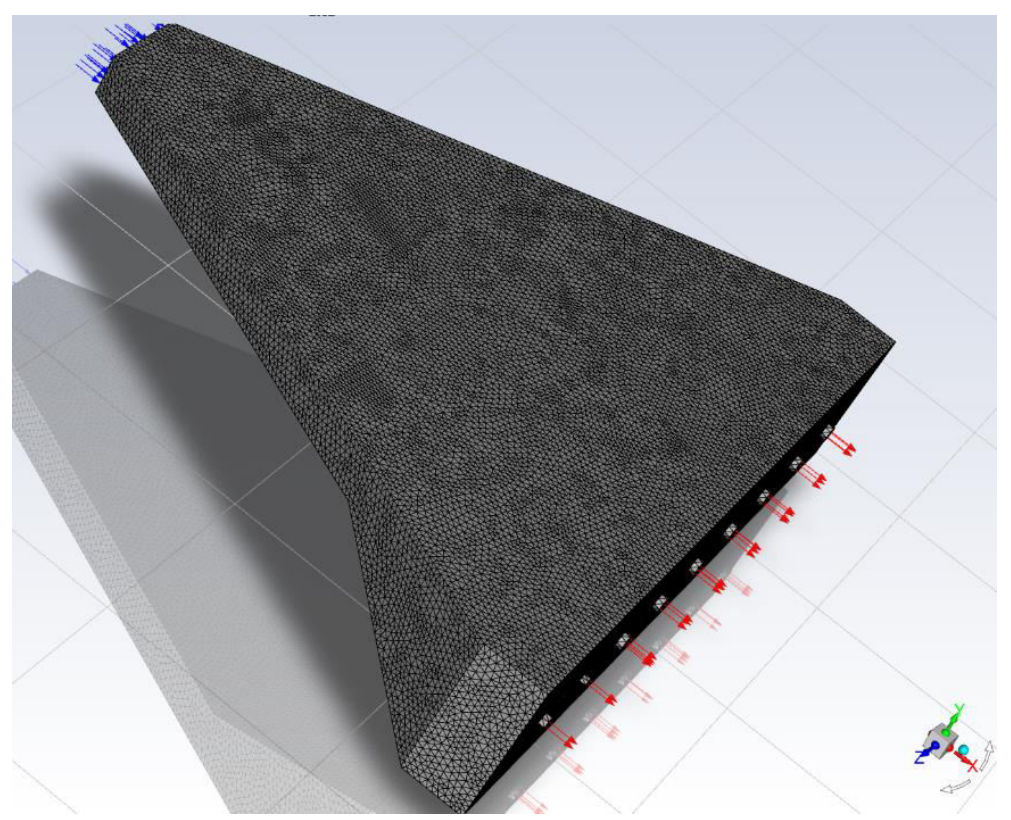

Figure 3 Grid division of the forebay of the pump station

The water diversion channel is set as the water inlet, and the boundary condition is the mixing velocity inlet, through which the whole water flow is controlled. According to the design flow rate and multiple measurements, the inlet velocity of the water and sand mixed phase is set as $0.4 \mathrm{~m} / \mathrm{s}$ because the water flow carries sediment particles, and the main phase water velocity and secondary phase sediment are also $0.4 \mathrm{~m} / \mathrm{s}$. The outlet boundary condition is outflow, and the operation mode is set to fully open 9 pumps (Including 7 large pumps, 1 small pump and 1 standby pump). Among them, the single flow rate of the large pump is $1.82 \mathrm{~m}^{3} / \mathrm{s}$, the single flow rate of the small pump is $0.52 \mathrm{~m}^{3} / \mathrm{s}$, and the single flow rate of the standby pump is $1.0 \mathrm{~m}^{3} / \mathrm{s}$. According to each outlet flow, the converted percentage of outlet water volume is 0.128 for 1-7 large pumps, 0.036 for 8 small pumps and 0.068 for 9 standby pumps. Because the fluid is stationary on the solid wall, the boundary of the entire flow region of the sediment two-phase flow is set as no slip constraint except for the inlet and outlet.

SIMPLEC improves the simple algorithm to speed up the convergence speed. When the velocity and flow do not satisfy the momentum equation, the SIMPLEC algorithm repeatedly calculates until the equilibrium condition is satisfied and adopt the second-order upwind scheme with better applicability. The relaxation factor takes the default value according to the SIMPLEC algorithm, and the number of calculated steps is 100,000 . The calculation accuracy sets the $\mathrm{x}, \mathrm{y}$ and $\mathrm{z}$ directions of the sediment and water as $10^{-4}$.

The standard $k-\varepsilon$ model, RNG $k-\varepsilon$ model and realizable $k-\varepsilon$ model were used in this calculation. The realizable $k-\varepsilon$ model was selected as the best match with the actual project flow pattern after many comparisons.

\section{Simulation analysis and discussion}

\subsection{Three-dimensional flow and sediment concentration analysis}

The two-phase water sand flow in the forebay of the pump station is numerically simulated based on the mixture model. In the model calculation, the flow pattern is analyzed after 100,000 
iterative steps, as shown in Figure 4.

Sand. Volume Fraction
Volume Rendering 1
\begin{tabular}{|l}
$6.292 \mathrm{e}-01$ \\
$5.611 \mathrm{e}-01$ \\
$4.929 \mathrm{e}-01$ \\
$4.248 \mathrm{e}-01$ \\
$3.566 \mathrm{e}-01$ \\
$-2.884 \mathrm{e}-01$ \\
$2.203 \mathrm{e}-01$ \\
$1.521 \mathrm{e}-01$ \\
$8.398 \mathrm{e}-02$ \\
$1.582 \mathrm{e}-02$
\end{tabular}
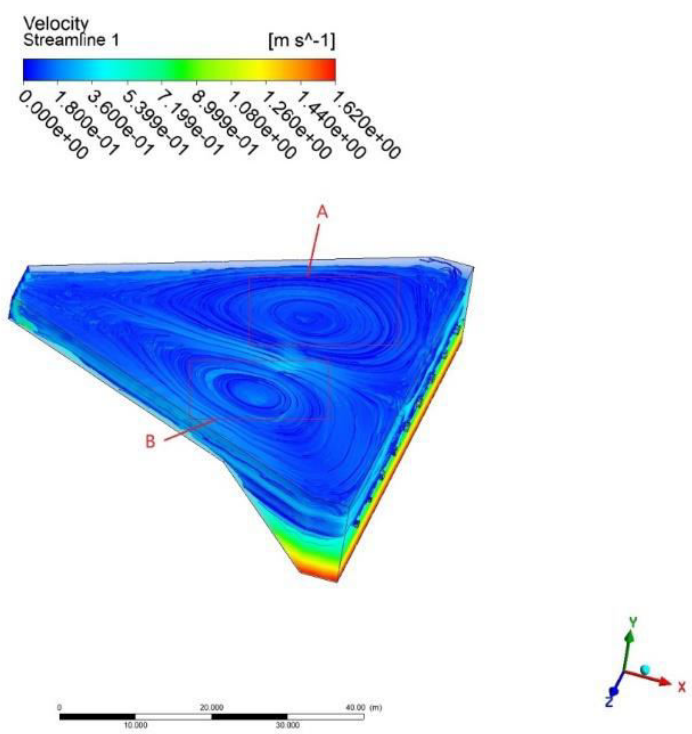

Figure 4 Flow line and sediment volume fraction in the forebay

The streamline forms two large vortices, A and B, on both sides of the forebay, and the whirling area of vortex A close to the large pump (Nos. 1 to 4 ) is larger than that of vortex B close to the small pump and standby pump (Nos. 6 to 9). The flow pattern is relatively stable at the center, and it flows directly from the inlet to the No. 5 pumping station. From bottom to top, as shown in Figure 5, two large vortices, $\mathrm{C}$ and $\mathrm{D}$, and some complex small vortices are generated at the inlet tank with a sloping bottom, but vortices $\mathrm{C}$ and $\mathrm{D}$ are slightly smaller than those of $\mathrm{A}$ and $\mathrm{B}$, and the whirling area of vortex $\mathrm{C}$ close to the large pump is smaller than that of vortex D close to the small pump. Local analysis is conducted for four large vortices, as shown in Figure 6. Lines $\mathrm{a}, \mathrm{b}$ and $\mathrm{c}$ correspond to the depth of the forebay at $3 \mathrm{~m}$, and lines $\mathrm{G}$ and $\mathrm{H}$ correspond to the inlet depth of $4.4 \mathrm{~m}$. Line a passes through vortex A and vortex C (upper surface), line b flows directly from the inlet to pumping station No. 5, and line c passes through vortex B and vortex D (upper surface). Figure 7 shows that the three line velocities change. The velocity of line $b$ in the middle area with a good flow pattern is higher, while the velocities of lines a and $\mathrm{c}$ in the area with a poor flow pattern are lower, and the velocities of lines a and c are basically symmetrical. At the same time, the flow velocity of this large vortex is basically maintained at approximately $0.15 \mathrm{~m} / \mathrm{s}$, and the velocity increase is approximately $0.01 \mathrm{~m} / \mathrm{s}$. For lines $\mathrm{G}$ and $\mathrm{H}$, when they are at the centers of vortices $C$ and $D$, the flow velocity remains at approximately $0.2 \mathrm{~m} / \mathrm{s}$, and the flow velocities of the two sections are almost symmetrical. The flow velocity near the small vortex in the forebay of the pump station is greater than that of the large vortex, the proximity to the pump port has a great impact on the flow velocity, and the fluctuation amplitude of the flow velocity increases. 

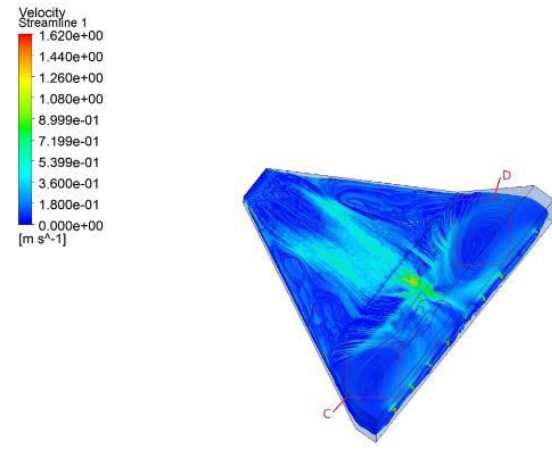

$K_{0}^{2}$

Figure 5 Flow line at the bottom of the forebay of the forebay

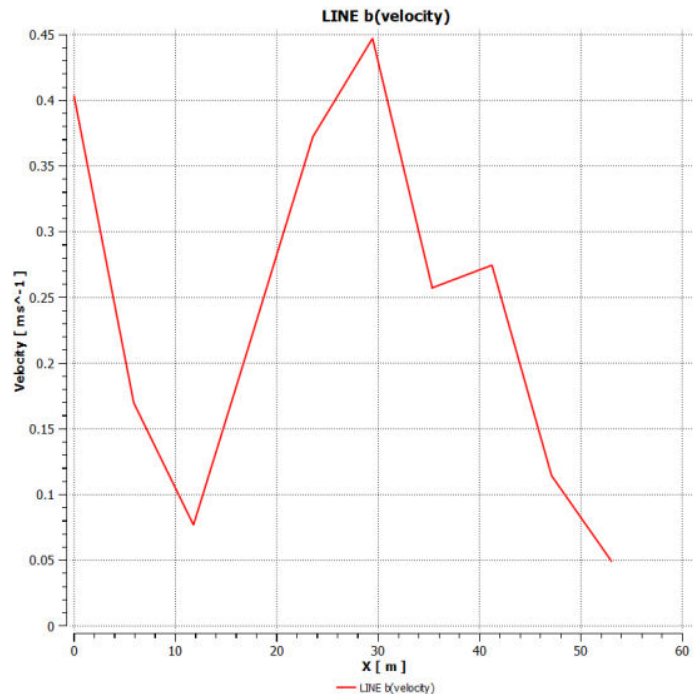

a. Line $b$ velocity variation

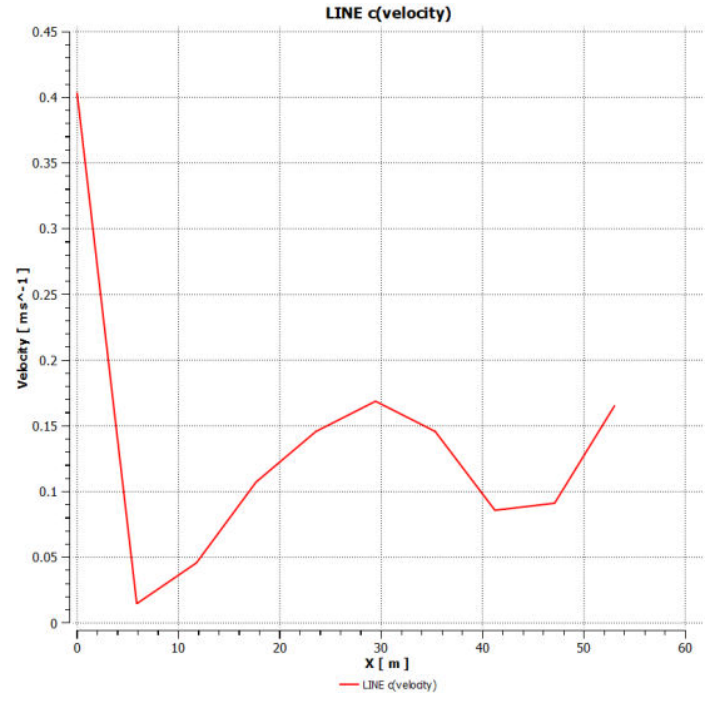

c. Line c velocity variation

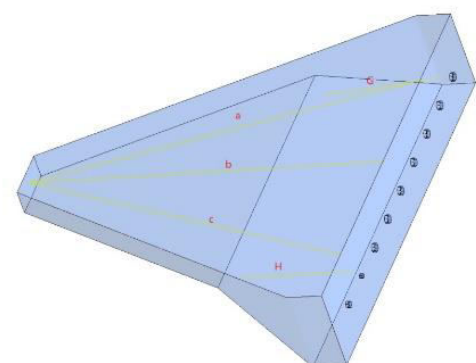

Figure 6 Location of the inner line

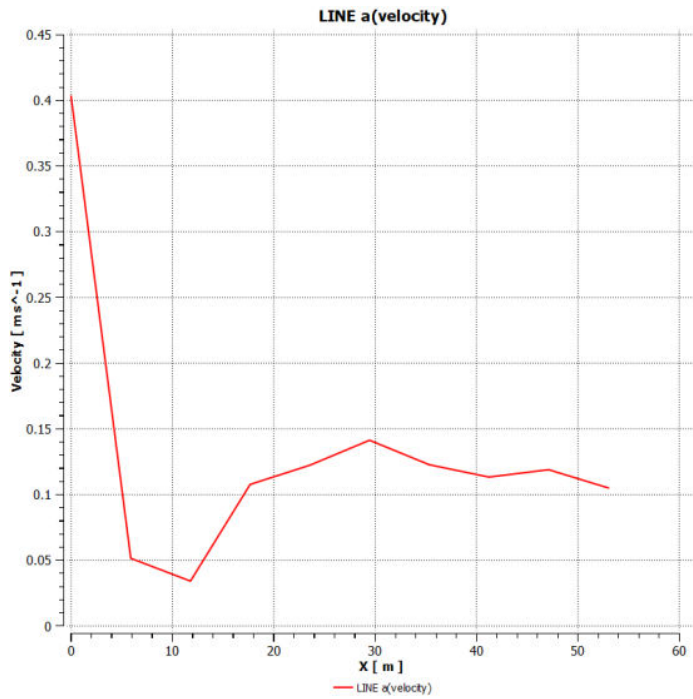

b. Line a velocity variation LINE G(velocity)

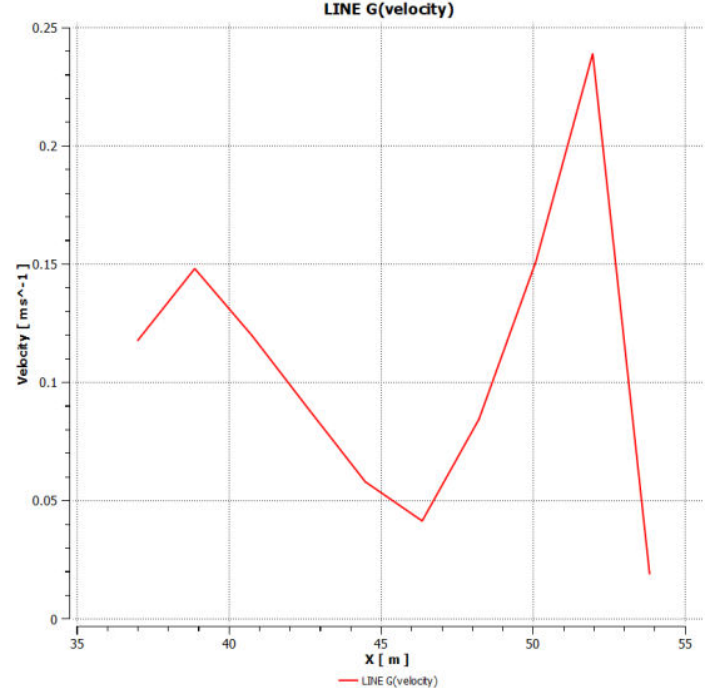

d. Line $\mathrm{G}$ velocity variation 


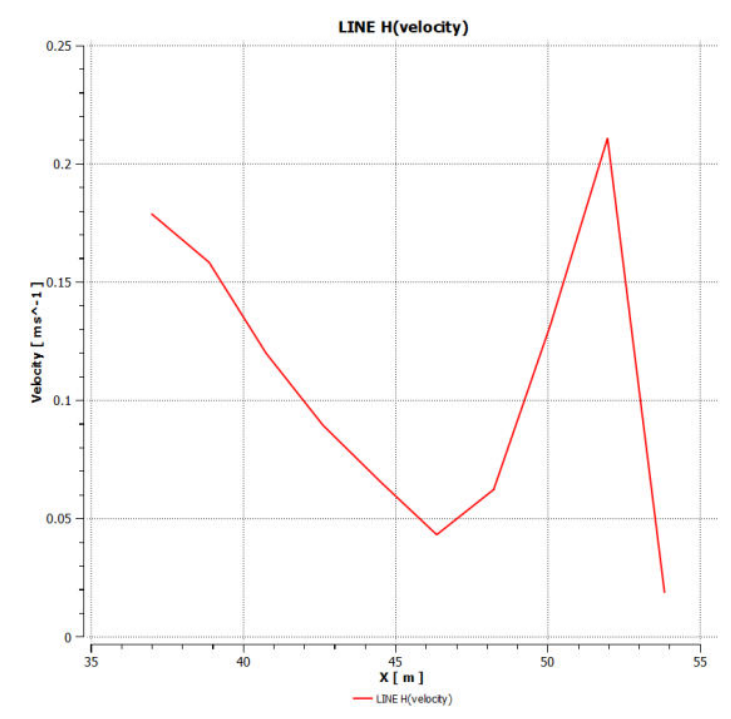

e. Line $\mathrm{H}$ velocity variation

Fig. 7 Flow velocity variation diagrams of individual lines

The volume fraction of the secondary phase sand is obtained according to equation (15), as shown in Figure 8, which is the volume fraction content of secondary phase sand, and the sediment volume fraction is roughly divided into four grades: (1) The volume fraction at the bottom of the inlet tank is as high as 0.6295 , where the sedimentation is the largest, and a thicker silt layer formed. (2) The sediment volume fraction directly below vortices $\mathrm{C}$ and $\mathrm{D}$ is between 0.4 and 0.55. This part is a area with high sediment content, and there is almost no streamline distribution. Partial sedimentation has formed. (3) Directly below vortices A and B, the sediment volume fraction is approximately 0.2 , the streamline distribution is sparse, and slight sediment deposition has formed. (4) The sediment volume content in the water flow area is approximately 0.015. At this time, the flow pattern of the sediment in the water is roughly the same as the flow line of the water, and there is no obvious sedimentation, but due to the change in the flow pattern and the influence of gravity, it moves and stacks to reform the original silt. The sediment volume fraction content can be used only to preliminarily determine the location of sedimentation, and it is necessary to analyze and study the specific sedimentation situation in each section.

Sand. Volume Fraction
Volume Rendering 1
\begin{tabular}{|l}
$6.292 \mathrm{e}-01$ \\
$5.611 \mathrm{e}-01$ \\
$4.929 \mathrm{e}-01$ \\
$4.248 \mathrm{e}-01$ \\
$3.566 \mathrm{e}-01$ \\
$2.884 \mathrm{e}-01$ \\
$2.203 \mathrm{e}-01$ \\
$1.521 \mathrm{e}-01$ \\
$8.398 \mathrm{e}-02$ \\
$1.582 \mathrm{e}-02$
\end{tabular}
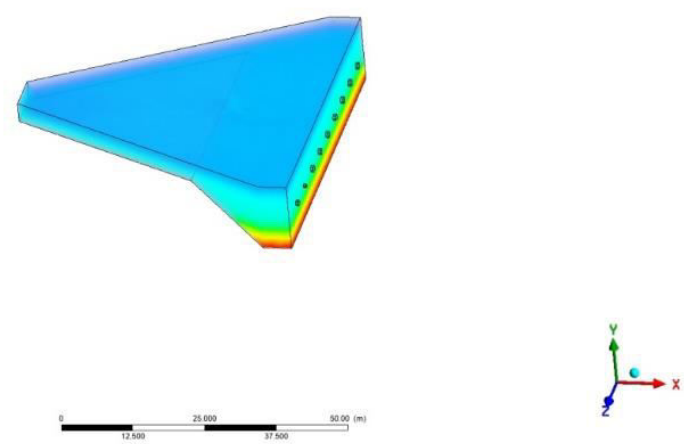

Figure 8 Sand volume fraction content 
Analysis of the reasons for the A, B, C, and D vortex areas: (1) Defects in the design of the forebay is the first reason. Due to the small diffusion angle and insufficient length of the diffusion section during the design of the front pool, the two-phase water-sand flow from the diversion channel pushes directly against the back wall in the direction of pump station No. 5; the large inertia of the flow results in two vortices, A and B, in the forebay. (2) Due to the large water flow and inertia in the mainstream area, pump No. 5 has no time to cause the flow to diffuse regardless of its pumping power. At this time, the reaction force of the rear wall on the water flow intensifies vortices A and B. (3) Due to the slope of the wall between the forebay and the inlet tank, the reaction force generated on the rear wall bounces back again, resulting in vortices $\mathrm{C}$ and $\mathrm{D}$. The continuous reaction force of the sloping wall leads to the continuous reduction in the flow velocity of vortices $C$ and D. (4) The complex flow pattern and the different pumping powers of pumps 1-9 make it impossible for vortices $\mathrm{A}$ and $\mathrm{B}, \mathrm{C}$ and $\mathrm{D}$ to be completely symmetrical.

Therefore, according to the above analysis, the following measures can be taken at the inlet forebay of the forward pump station to eliminate or reduce the reflux area: 1. Increase the length of the diffusion section by an appropriate amount when the pump station forebay is newly built. 2 . When the inlet flow velocity is greater than $0.4 \mathrm{~m} / \mathrm{s}$, increase the diffusion angle by an appropriate amount within the allowable specification. 3. On the rear wall of the forebay, add an energy dissipation device between pumps 1-4 and pumps 6-9 to reduce the inertial force brought by the main flow area and reduce the A and B vortex areas. 4. Add rectification or energy dissipation measures on the sloped sidewall to weaken the backflow area in the inlet tank.

\subsection{Analysis of the flow field and sediment content according to a two-dimensional top view}

Figure 9 shows the horizontal bottom of the forebay as section a-a, extending upward for 1,2 and $3 \mathrm{~m}$, and sections b-b, c-c and d-d. According to the analysis of section a-a, there are basically symmetrical sediment areas on both sides of the forebay. According to the comparison of vortices $\mathrm{A}$ and $\mathrm{B}$ in the previous section, the top view shows that the area with a high sediment volume fraction in area a with large vortices A is smaller than that in area B with small vortices, and the area difference is about $5 \mathrm{~m}^{2}$. According to the vector diagram of section a-a, when the sediment volume fraction is more than 0.245 , there is no streamline distribution. The research and analysis show that a very large sediment deposition has formed in this area. The vector diagrams of d-d, c-c and $\mathrm{b}-\mathrm{b}$ show that the vortex first increased and then decreased from top to bottom, the flow velocity continued to decrease, the flow pattern was extremely disordered, the sediment volume fraction content increased in turn, and a slight sediment deposition formed on the c-c section. At this time, the sediment volume fraction content reached 0.211 . Therefore, the sediment deposition height was approximately between 2.0 and $2.2 \mathrm{~m}$. 

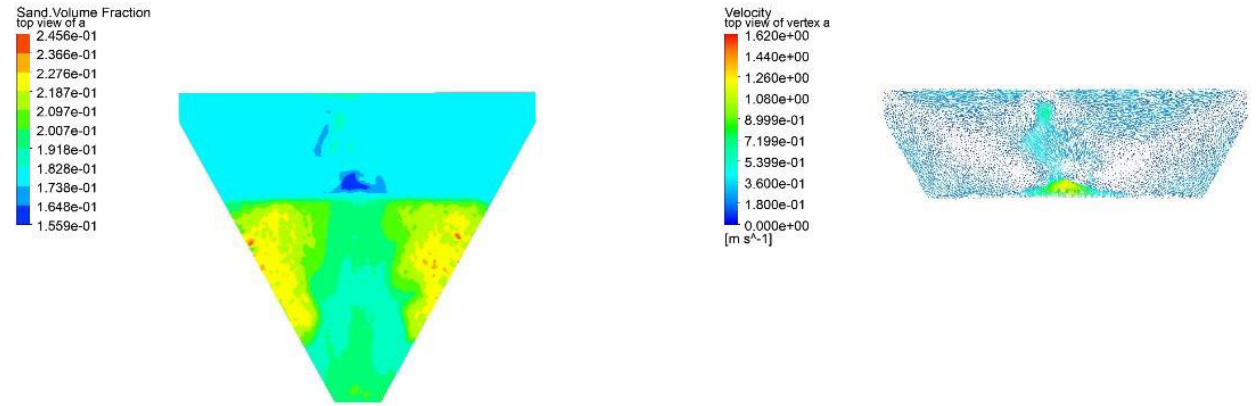

$\stackrel{x+1}{-1}$

a-a Sand volume fraction content

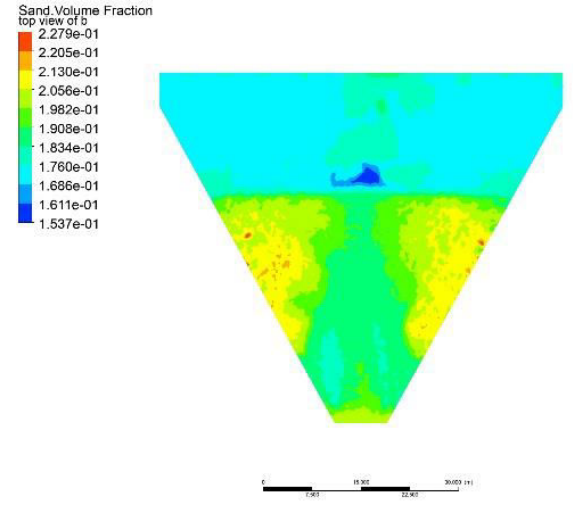

b-b Sand volume fraction content

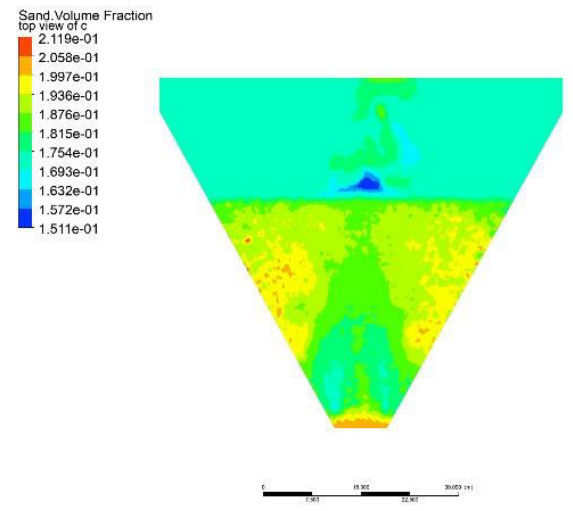

c-c Sand volume fraction content

$\stackrel{x}{\longrightarrow}=$

$\stackrel{x}{\longrightarrow}=$

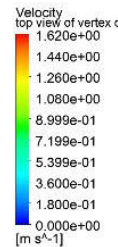

$\stackrel{x}{\leftrightarrow}$
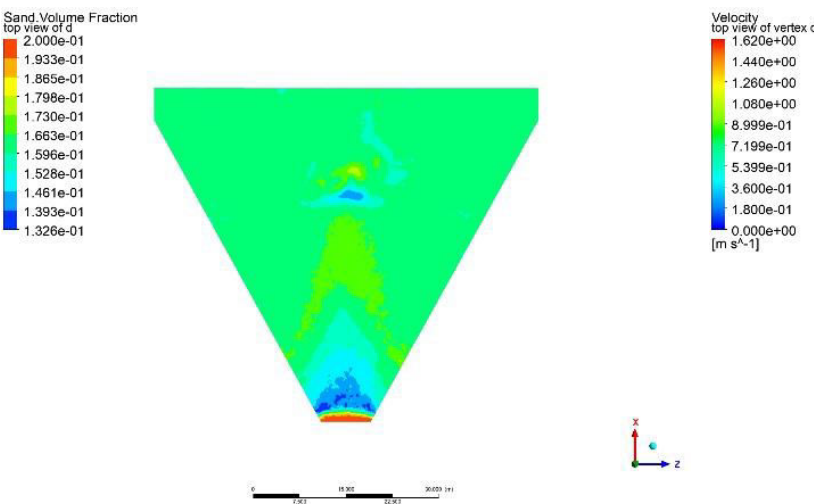

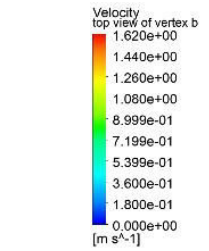

a-a Vector diagram

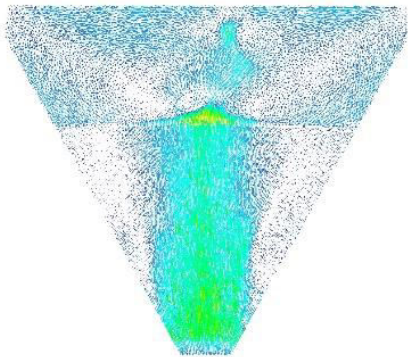

$\stackrel{x}{\stackrel{L}{\infty}=}$

b-b Vector diagram

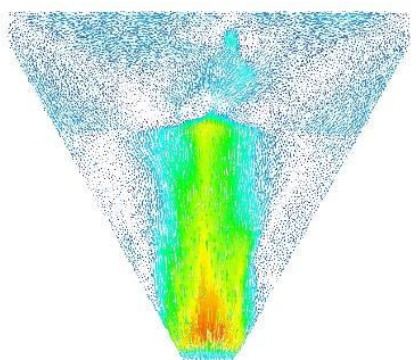

$\stackrel{x}{\longrightarrow}=$

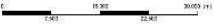

c-c Vector diagram
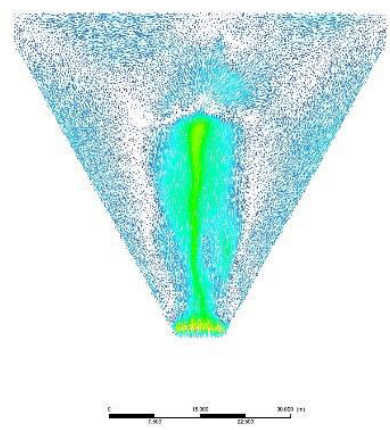

$\stackrel{+}{1}$ 
Figure 9 Sand volume fraction content and vector diagram of each section in the forebay

Figure 10 shows a sloped inlet tank with section a-a as the zero interface and sections e-e, f-f, $\mathrm{g}-\mathrm{g}$, and $\mathrm{h}-\mathrm{h}$ at 2, 4, and $6 \mathrm{~m}$ and the bottom, respectively. Vortices are generated in the vector diagrams from e-e to $\mathrm{g}$-g, and from top to bottom, the vortex size changes from large to small. In section e-e with the largest vortex, the sand volume fraction is higher in the middle, and the sand volume fraction on both sides reaches 0.2 . In section $\mathrm{f}-\mathrm{f}$, the sand volume fraction content is lower in the middle and higher on both sides. In the g-g section, the sediment volume fraction content is basically evenly distributed, and it can reach 0.421 . The depth of the vortex is approximately 2 to $3 \mathrm{~m}$. Because of the action of the roundabout, there is basically no streamline distribution or the streamline distribution is extremely dispersed in the $\mathrm{g}-\mathrm{g}$ and $\mathrm{h}-\mathrm{h}$ sections; at this time, the force of gravity on the sediment is greater than the transverse velocity, and the solids move to the bottom area to form a thicker sediment layer. The height of the siltation layer is about $2.0 \mathrm{~m} \sim 2.5 \mathrm{~m}$ above the $g$-g section and below the f-f section, and the maximum sediment volume fraction can reach 0.6295 .
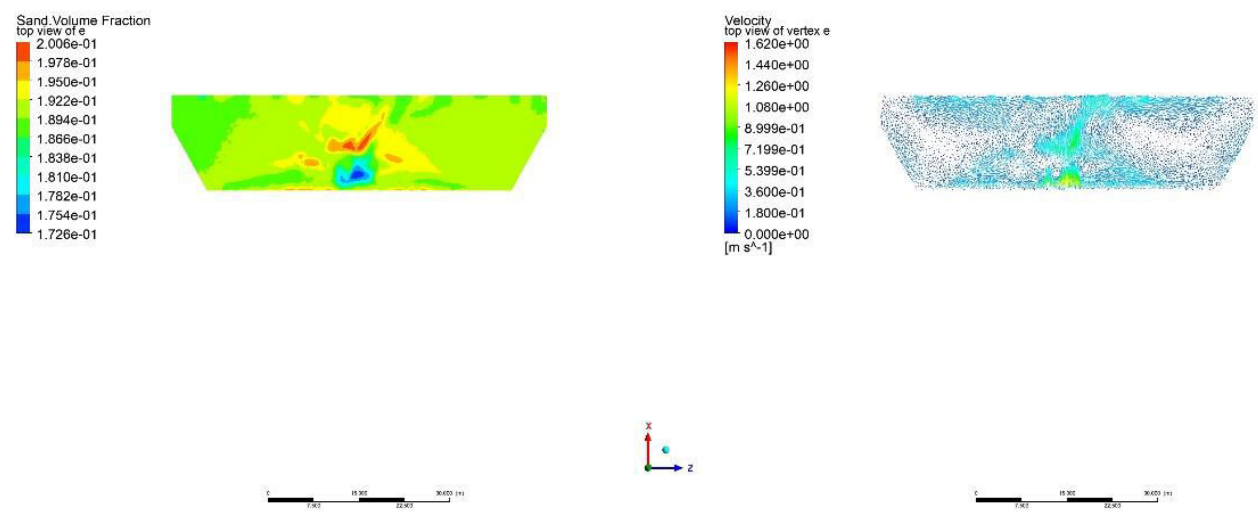

$\stackrel{x}{\longrightarrow}$

e-e Sand volume fraction content
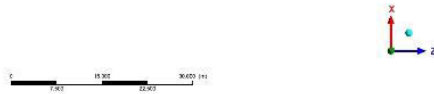

e-e Vector diagram
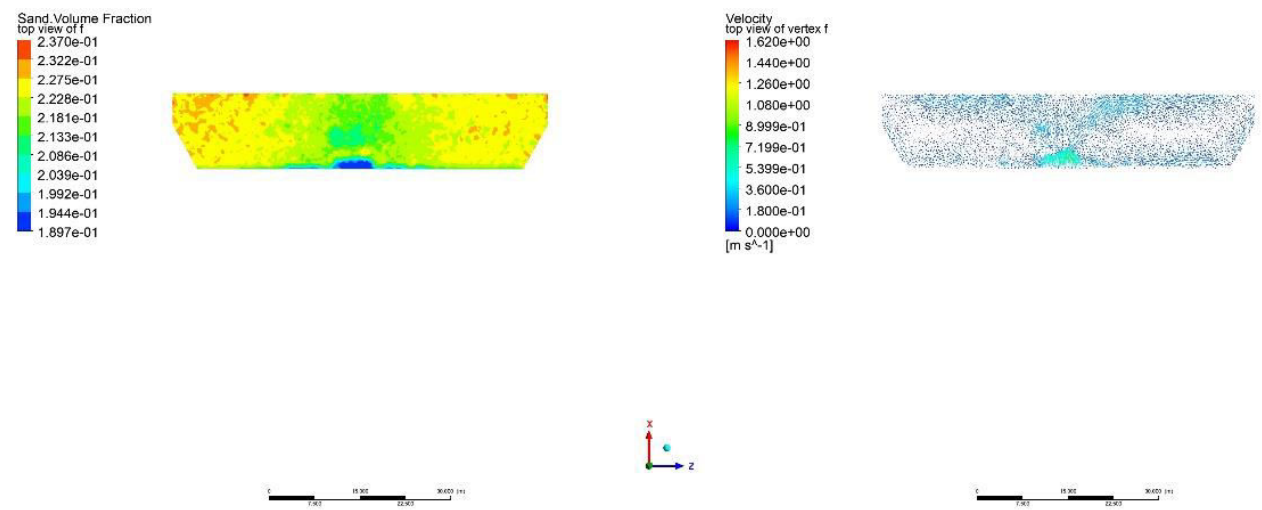

$\stackrel{x}{\longrightarrow}$

f-f Sand volume fraction content

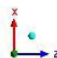

f-f Vector diagram 


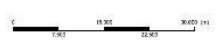

g-g Sand volume fraction content

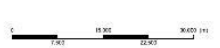

g-g V diagram
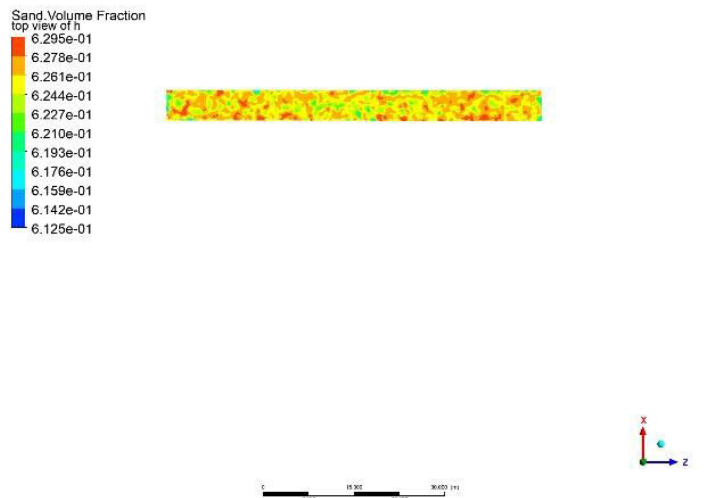

h-h Sand volume fraction content

Figure 10 Sediment volume fraction content and vector diagram shown in the top view of each section of the inlet tank

Therefore, the area and height of the large sedimentation can be determined from the top view. For the forebay of the pump station without various measures, the silted sediment worsens the flow pattern of the forebay and inlet tank, resulting in the low operational efficiency of the pump station and the lack of a timely supply of agricultural irrigation. At this time, the intermediate pumps (Nos. 4, 5 and 6) can be opened to temporarily improve the operational efficiency of the pump, and staggered peak irrigation should be implemented to alleviate the operational pressure of the pump station and the harm caused by sediment deposition.

\subsection{Analysis of two-dimensional longitudinal and cross-sectional views}

To make the research results universal, the longitudinal view focuses on analyzing the flow pattern of the inlet tank, and the cross-sectional view focuses on analyzing the flow pattern of the forebay. Taking the center of pumping stations Nos. 1 to 9 as the base point, Figure 11 shows longitudinal diagrams $1-1,1-2,1-3,1-4,1-5,1-6,1-7,1-8$, and 1-9. According to the vector diagram analysis of pumps No. 1 to No. 4, the flow pattern below the suction pipe is extremely turbulent; the flow pattern has not only areas with different degrees of swirling but also relatively sparse flow lines, and the flow pattern above the suction pipe is relatively stable. The main reasons are as follows: First, there is already a bad flow pattern in the forebay, and the bad flow pattern is exacerbated twice in the inlet pool. Second, due to the positive correlation between the sediment transport rate and flow velocity, in this backflow area, the adverse flow pattern makes the flow velocity lower than the minimum sediment carrying velocity, and the sediment density is large. 
Under the influence of gravity, it intensifies the sediment backflow to the bottom and continues to settle, making the sediment in the inlet tanks of pumps Nos. 1-4 heavier. According to the diagram of sediment volume fraction content, the sedimentation degree is basically $4 \mathrm{~m}$ at the edge of the suction pipe, there is no obvious streamline distribution at the bottom $2 \mathrm{~m}$, and the sediment is heavily silted.
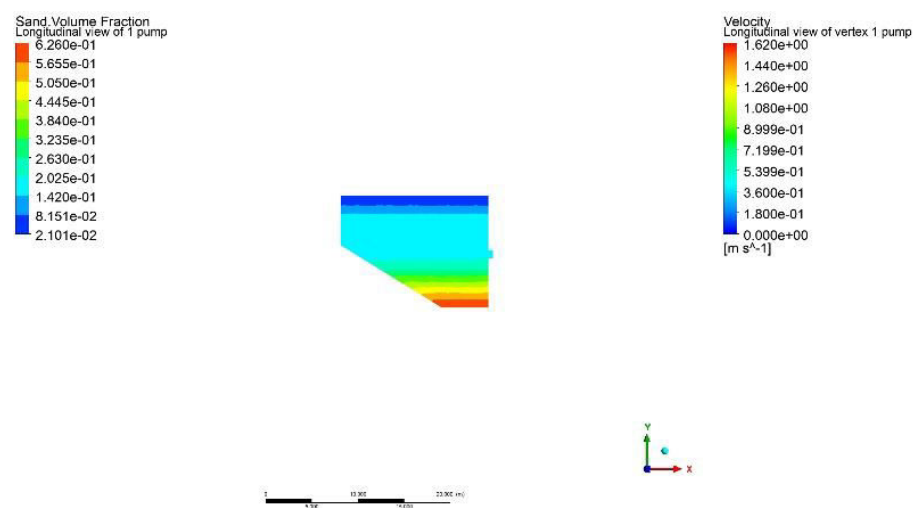

1-1 Sand volume fraction content
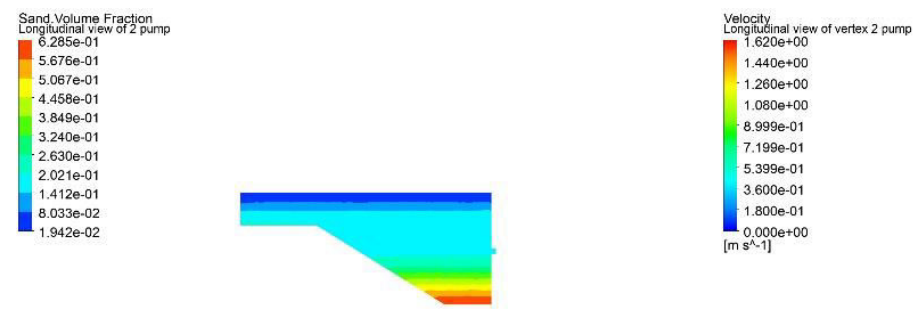

$\stackrel{\circ}{\leftrightarrow}$

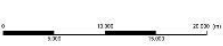

$\stackrel{.}{\longleftrightarrow}$

1-1 Vector diagram
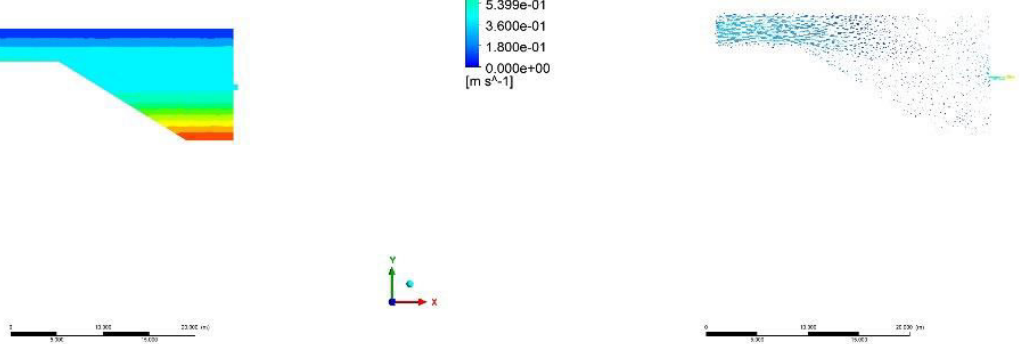

$\stackrel{.}{\longrightarrow}$

1-2 Sand volume fraction content

1-2 Vector diagram
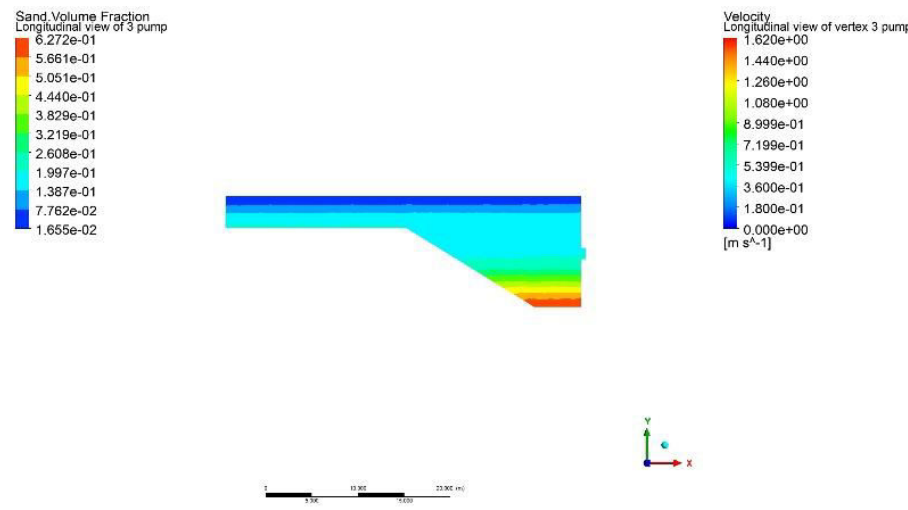

$\stackrel{.}{\longleftrightarrow}$

1-3 Sand volume fraction content

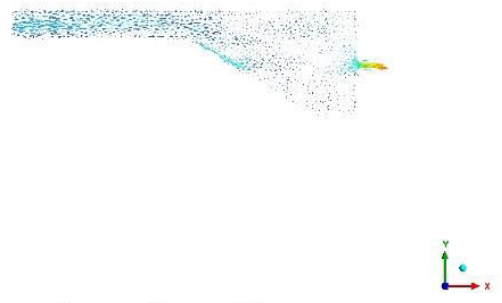

1-3 Vector diagram 

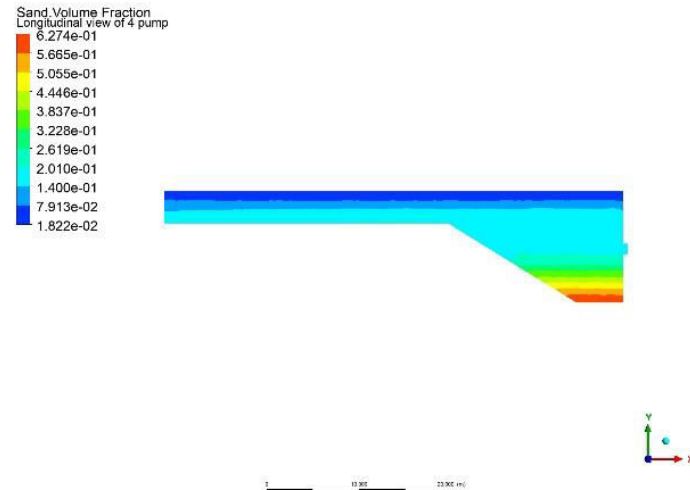

1-4 Sand volume fraction content
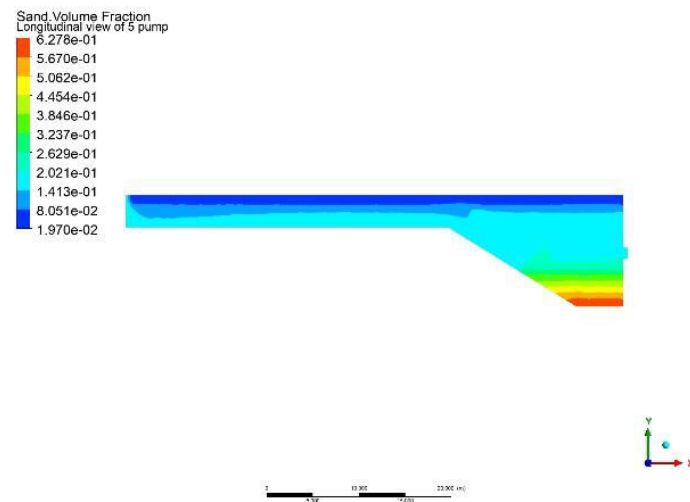

1-5 Sand volume fraction content
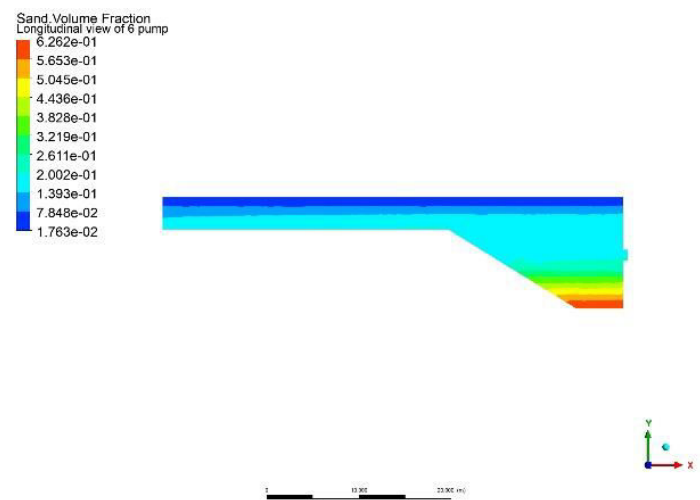

1-6 Sand volume fraction content
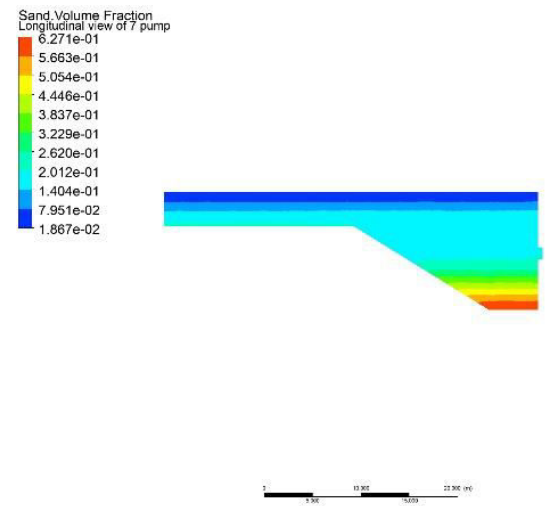

1-7 Sand volume fraction content

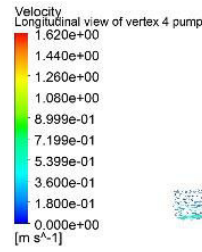

$\stackrel{\leftrightarrow}{\rightarrow}$

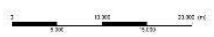

$\stackrel{\leftrightarrow}{\longleftrightarrow}$

1-4 Vector diagram

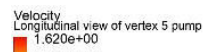

$1.6200+00$
$1.440 \mathrm{e}+00$

$1.260 \mathrm{e}+00$

$.0800+0$

.

$5.399 \mathrm{e}-01$

$3.600 \mathrm{e}-01$
$1.800 \mathrm{e}-01$ $\stackrel{\lfloor\cdot \circ}{\longleftrightarrow}$

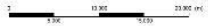

1-5 Vector diagram

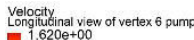

$1.620 \mathrm{e}+00$
$1.440 \mathrm{e}+00$

$-1.2600+00$

$1.0800+00$

$8.999 \mathrm{e}-01$
$7.1998-01$

$-5.399 \mathrm{e}-0$

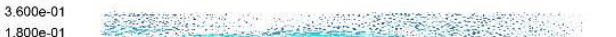

$1.8000-01$

Imsilit

$\leftrightarrow x$

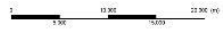

$\stackrel{\lfloor. .}{\longleftrightarrow}$

1-6 Vector diagram

$\stackrel{\leftrightarrow}{\leftrightarrow} x$ $\stackrel{\leftrightarrow}{\longleftrightarrow}$

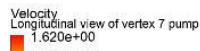

$1.620 \mathrm{e}+00$
$1.440 \mathrm{e}+00$

$-1.260 \mathrm{e}+00$

$1.0800+00$

8.999e- 01

$7.199 \mathrm{e}-0$
$5.399 \mathrm{e}-0.0$
3.0000

. $6000 \cdot 0$

$\left[\begin{array}{ll}0.0000 e+00 \\ 0 \\ \left.s^{0}-1\right]\end{array}\right.$ 

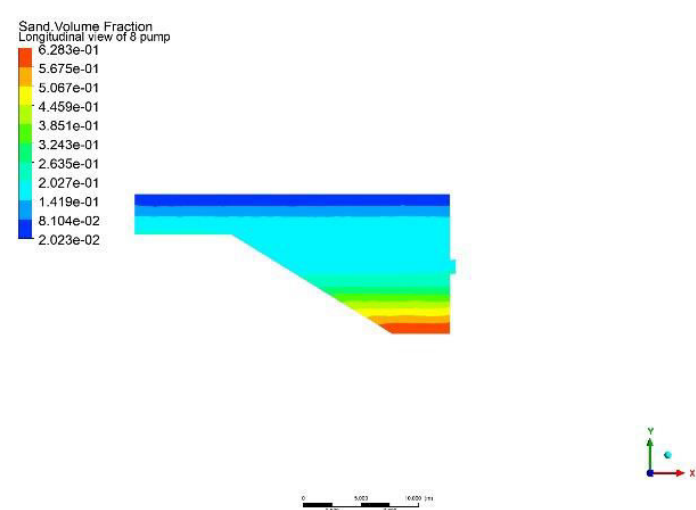

1-8 Sand volume fraction content
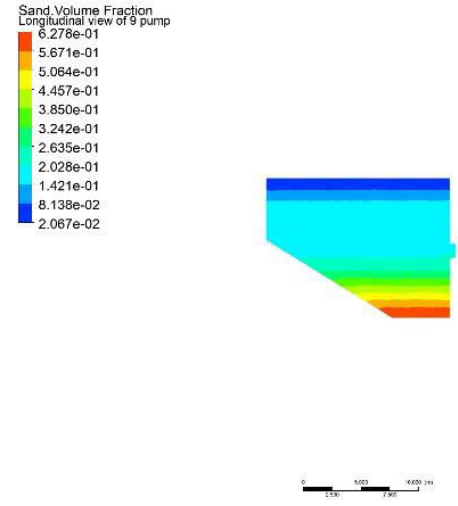

$\stackrel{\circ}{\longleftrightarrow}$

1-9 Sand volume fraction content
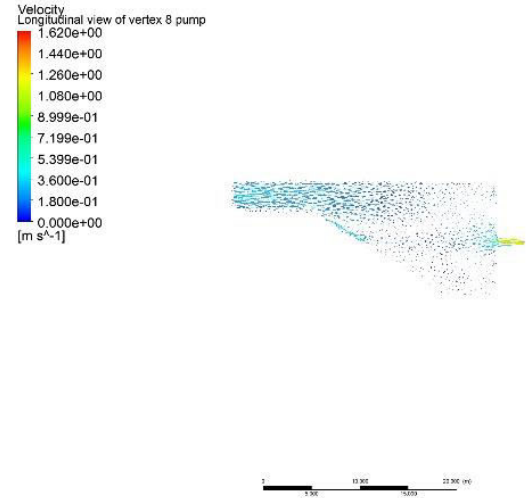

$\stackrel{.}{\longleftrightarrow}$

1-8 Vector diagram
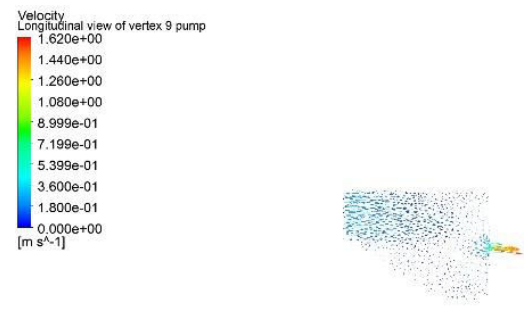

$\stackrel{.}{\longleftrightarrow}$

1-9 Vector diagram

Figure 11 Sand volume fraction contents and vector diagrams in the longitudinal plane of each

$$
\text { pump station }
$$

Compared with pump stations No. 6 to No. 9, the sediment settles at the bottom of the intake tank due to the action of vortices B and D. The flow pattern of pump No. 5 is good, but large sediment deposits form at the bottom. According to the comparison of the nine longitudinal planes, the silt layer basically presents a linear relationship; the silt layer from pumps No. 1 and No. 9 into the inlet tank is the smallest, while the sediment volume fractions of pumps Nos. 5 to 8 and pumps Nos. 2 to 5 first decrease and then increase. The main reason for this phenomenon is the change in vortex degree and the presence of the centers of vortices $\mathrm{C}$ and $\mathrm{D}$ directly below pumps No. 3 and No. 7. The sediment in this area moves slowly to both sides due to the vortex. The No. 1 and No. 9 pumps are at the edge of the sediment. At this time, the force of the vortex on them is basically offset, so the sediment deposition at this position is appropriately low.

Analysis of the cross sections of the flow field and sediment content was performed on sections n-n, m-m, p-p, q-q, y-y, z-z and w-w at distances of 5, 10, 15, 20, 25, 30 and $35 \mathrm{~m}$ after the inlet, as shown in Figure 12. From the analysis of the n-n plane, when the calculated height from the bottom is less than $0.4 \mathrm{~m}$, the sediment volume fraction content is the highest and shows a trend from low on both sides to high in the middle. Through vector diagram analysis, a small range and a variety of complex small vortices were generated, mainly on both sides of the flow field. However, from the $\mathrm{m}-\mathrm{m}$ to the $\mathrm{w}-\mathrm{w}$ plane, the vector diagram analysis shows that a variety of complex small vortices gradually form large vortices, and these vortices first increase and then decrease, reaching their maximum size between 10 and $15 \mathrm{~m}$ from the inlet, making the flow pattern in the forebay extremely disordered. Therefore, the content of the sediment volume 
fraction changes from high in the middle and low on both sides to high in the middle and low on both sides, and sediment deposition gradually moves to both sides of the forebay. According to the cross sections, the flow state is good, and the backflow vortex is small before $5 \mathrm{~m}$ from the inlet of the forebay. However, when the distance from the inlet is from 5 to $20 \mathrm{~m}$, the flow pattern changes greatly, and the degree of the vortexes reduces the flow velocity of the sediment and water, causing the sediment to move continuously to the two sidewalls. Only the flow pattern in the middle area is slightly stable, and the streamline moves to the center of the section after the q-q plane.
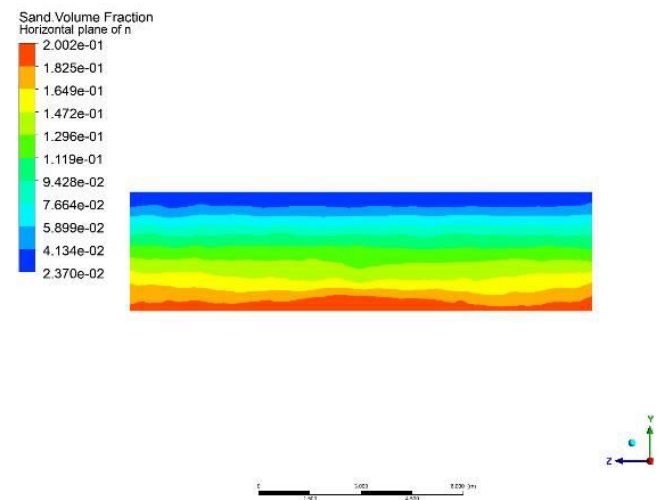

n-n Sand volume fraction content
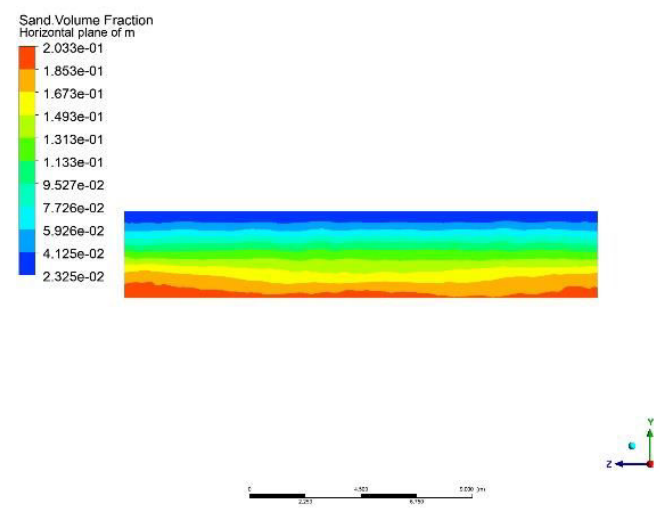

m-m Sand volume fraction content
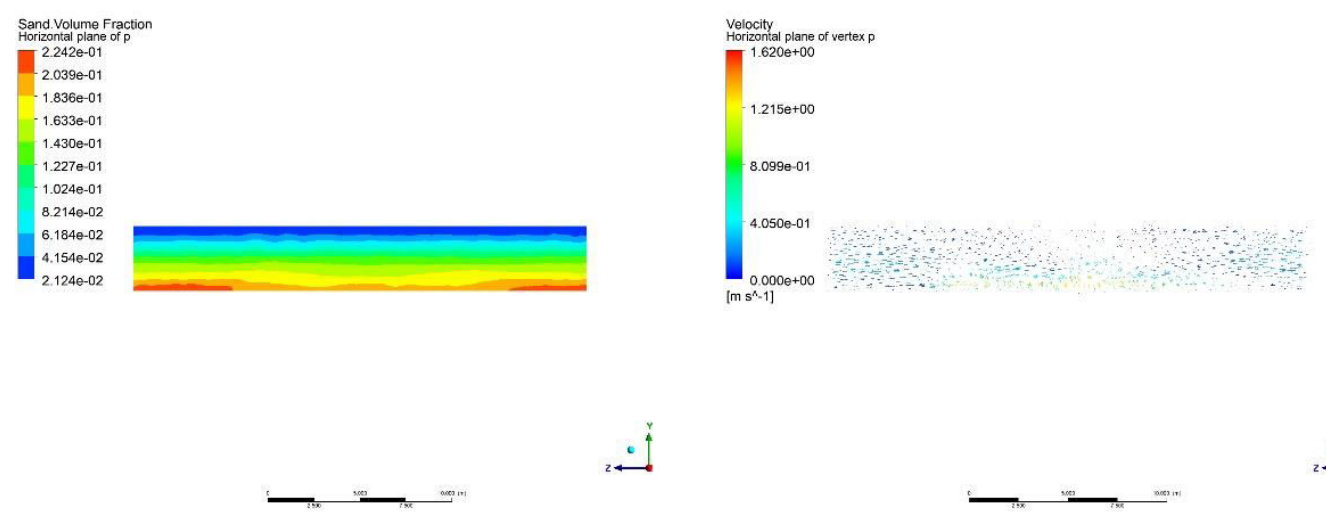

p-p Sand volume fraction content
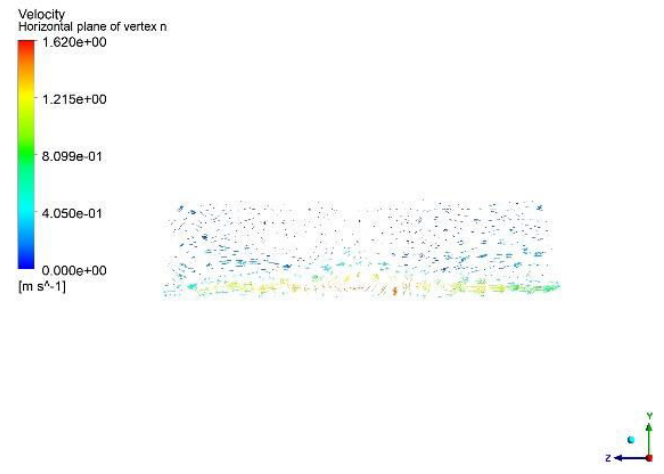

n-n Vector diagram
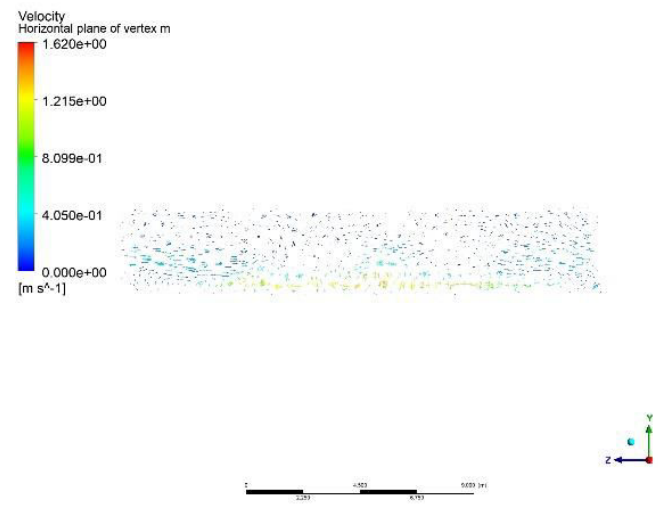

m-m Vector diagram 


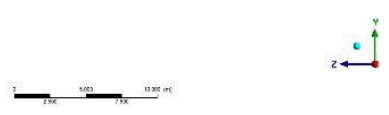

q-q Sand volume fraction content

$\stackrel{\circ}{\longleftarrow}$
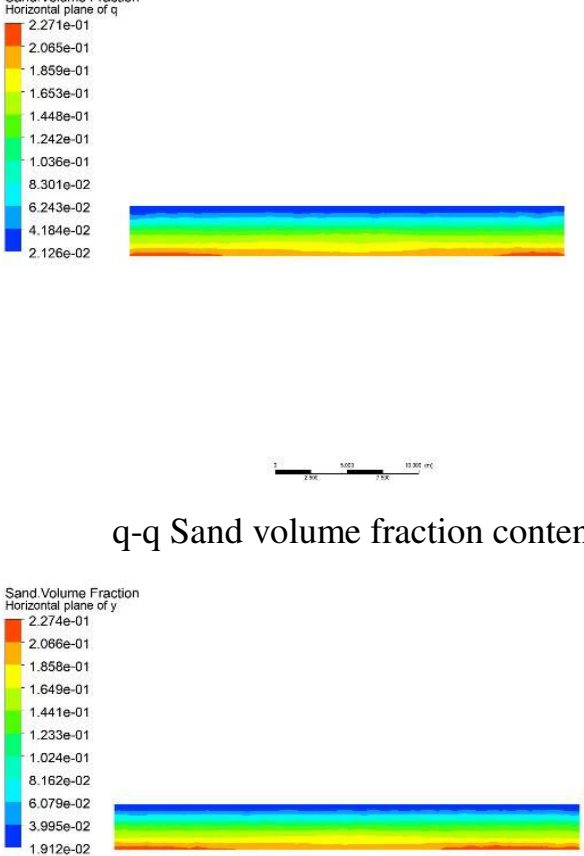

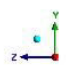

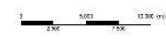

y-y Sand volume fraction content
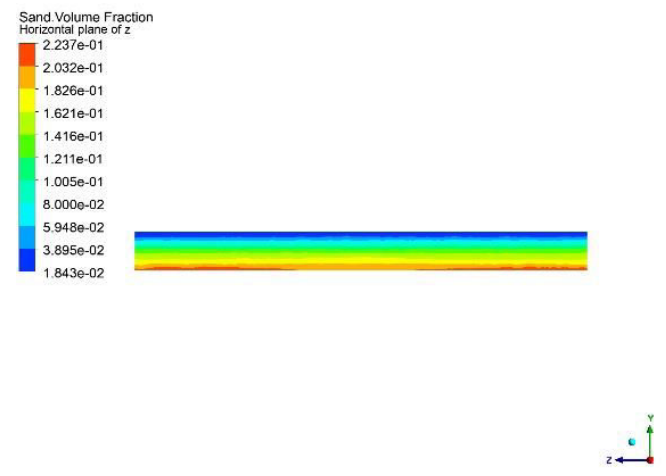

z-z Sand volume fraction content

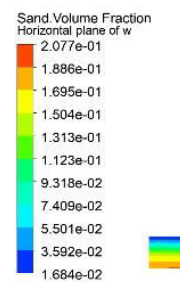

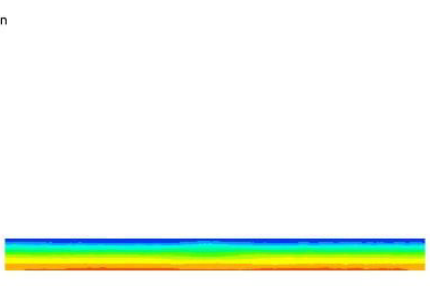

w-w Sand volume fraction content

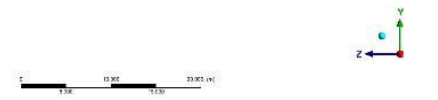

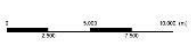

q-q Vector diagram

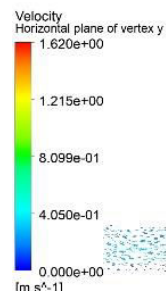

$-\ldots$

y-y Vector diagram
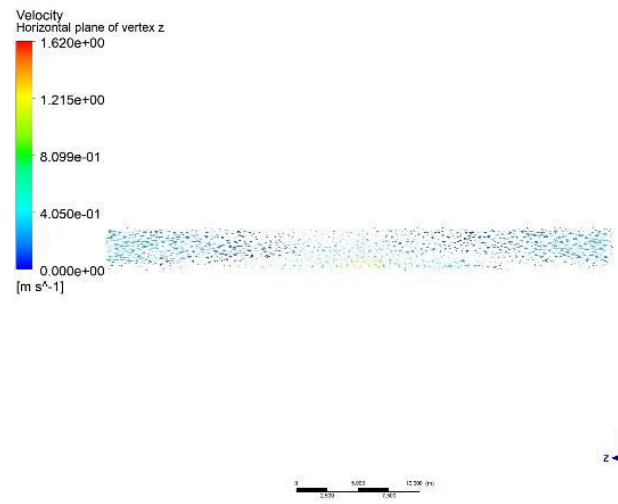

z-z Vector diagram

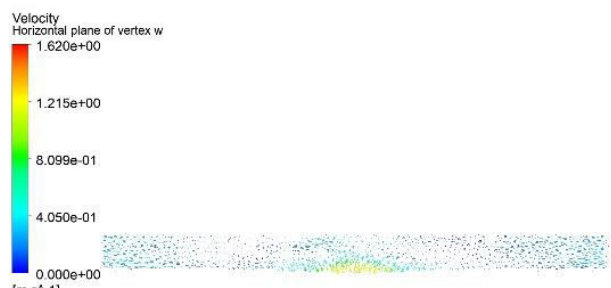

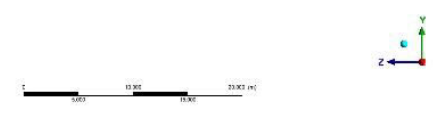

w-w Vector diagram 
Fig. 12 Sediment volume fraction content and vector diagram in the cross sections of each section of the forebay

The above analysis of the longitudinal planes shows that the severe sedimentation areas are basically the same as those shown in the top view, but the range of light sedimentation in the inlet tank is between 2 and $4 \mathrm{~m}$ in height. If the demand for agricultural irrigation is urgent and the inlet tank is silted, the vector diagram analysis proves that the intermediate pump should be properly started to alleviate the demand and temporarily improve the service efficiency of the pump station. In the actual project, some measures to improve the flow pattern are applied to the two sides between 1 and $5 \mathrm{~m}$ from the inlet of the forebay of the pump station to weaken the backflow in this area and provide good conditions for the pumping outlet of the pump station.

\section{Engineering project}

\subsection{Overview of irrigation area}

The Yanhuanding Yellow River common project is a large-scale electric water lifting project to solve the difficulty of supplying drinking water for humans and livestock in some areas of Shanxi, Gansu and Ningxia caused by the very uneven distribution of rainfall in the north and the many restrictions on agricultural irrigation. The project is located at the intersection of the Kushui River system in the Yellow River basin and the inland river basin of Yanchi. The project area is an inland location that is far from the coasts, with a typical continental climate and a mid-temperate arid zone. The annual average precipitation is $254.8 \mathrm{~mm}$, decreasing from south to north, with large interannual variation and uneven distribution within the year. It is mainly concentrated in the three months from July to September, accounting for $62 \%$ of the annual precipitation. The annual maximum precipitation is $586.8 \mathrm{~mm}$, the annual minimum is only $145.3 \mathrm{~mm}$, the annual range is more than 4 times the annual minimum, and the annual average evaporation is $2100 \mathrm{~mm}$.

This paper mainly studies and analyzes the second pump station (Wulipo pump station) in the project. The second pumping station belongs to a hilly landform unit, and the ground elevation is $1153 \sim 1158 \mathrm{~m}$. The area of the pumping station is a low and gentle hilly landform. After long-term denudation and deforestation, it presents a low undulating topography. The terrain is generally high in the southwest and low in the northeast. The topography of the site area is relatively flat and wide, and physical and geological features are not developed. The second pump station has a "straight side and forward water inlet" layout. The pump station is equipped with 9 pumps ( 7 large, 1 small and 1 standby), of which the standby pump is used specifically to supply water to the Wulipo irrigation area. The main buildings include inlet gates, forebays, pump houses, outlet pipes, outlet tanks, etc. An open split structure and lateral contraction style are adopted in the forebay. The forebay is divided into sloped sections and horizontal sections. The sloped section adopts a "U"-shape structure, with a length of $9.64 \mathrm{~m}$, width of $9.1 \mathrm{~m}$ and slope ratio of 1:4. The top elevation of the sidewall is $1155.8 \mathrm{~m}$, and the thickness is $0.5 \mathrm{~m}$. The top elevation of the bottom plate is from $1150.0 \mathrm{~m}$ to $1152.41 \mathrm{~m}$, and the thickness is $0.6 \mathrm{~m}$. The horizontal section adopts a split structure, the upstream sidewall adopts a cantilever reinforced concrete retaining wall, and the bottom plate adopts a reinforced concrete slab. For the pump house, a reinforced concrete dry chamber style is adopted. The main powerhouse has a building area of $1201.20 \mathrm{~m}^{2}$, a length of $85.80 \mathrm{~m}$, a width of $14.00 \mathrm{~m}$, a closed ring thickness of $0.6 \mathrm{~m}$, a bottom plate thickness 
of $0.8 \mathrm{~m}$, a reinforced concrete structure, and $0.25 \mathrm{~m}$ thick $\mathrm{C} 15$ concrete set at the top of the bottom plate. Figure 13 shows the geographic location of the area.

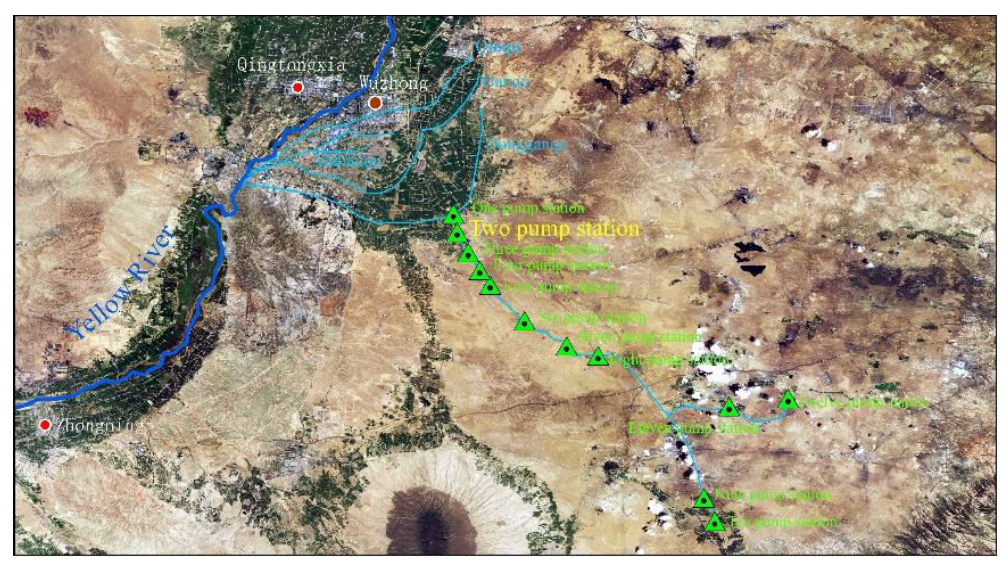

Fig. 13 Location map

4.2 Sediment deposition in the forebay of the pump station

According to the field investigation, as shown in Figure 14, there is a large amount of sediment deposition in the forward inlet forebay of the second pump station, and the degree of deposition extends to the inlet tank, which seriously affects the normal water intake of the pump. The silt layer thickness of the second pump station is measured and analyzed, as shown in Figure 15. The dotted line in the figure shows the area with large sediment deposition, with a height of approximately between 2.0 and $2.5 \mathrm{~m}$, and the solid line shows the area with moderate sediment deposition, with a height of approximately between 0.7 and $1.0 \mathrm{~m}$. The sediment in the forebay is basically symmetrical, but the sedimentation area near the large pump is less than that near the small pump, approximately between 3 and $5 \mathrm{~m}^{2}$. The degree of sediment deposition is consistent with the numerical simulation results. Therefore, the numerical simulations can provide reliable guidance and ways to improve engineering projects.

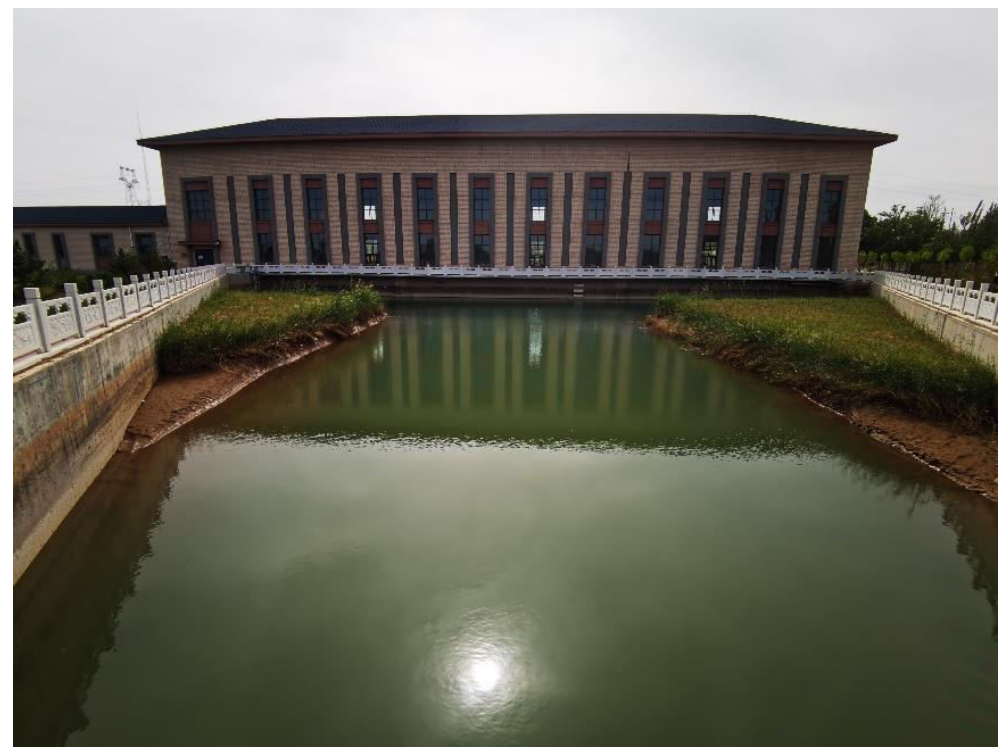

Fig. 14 Sediment deposition 


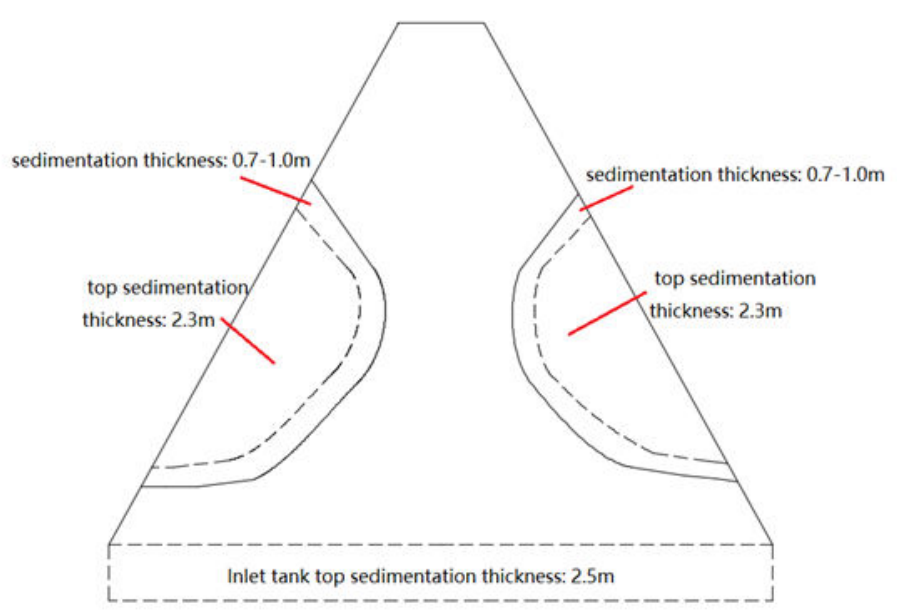

Fig. 15 Sediment deposition distribution

\section{Conclusions}

The problems associated with vortices and sediment deposition in the forebay of a forward inlet pump station affect the normal operation and safety of the pumping station. In this study, a numerical simulation is established based on the mixture model theory by Fluent, the two-phase water sand flow is analyzed, and the realizable $k-\varepsilon$ turbulence equation is used to solve this problem. The numerical simulation results are basically consistent with actual engineering projects, and the flow patterns and sand volume fraction contents in different sections are analyzed and verified. The research shows that the high sediment concentration of the Yellow River is the fundamental cause of sediment deposition, but the disorder of the flow pattern in the forebay is a design defect. The following conclusions can provide a reference for the construction or reconstruction of the forebay of the forward inlet pump station in the future:

(1) Through the analysis of the three-dimensional flow patterns and sediment contents, when the forebay of the pump station is newly built, the length of the diffusion section can be increased by an appropriate amount, and when the inlet velocity is greater than $0.4 \mathrm{~m} / \mathrm{s}$, the diffusion angle can be increased by an appropriate amount to reduce the scope of the raceway.

(2) During the reconstruction of the pump station, energy dissipation devices can be set on both sides of the rear sidewall to reduce the inertial force brought by the mainstream. Rectification or energy dissipation devices can be added between the sloped inlet tank and the forebay to reduce the force generated by rebound to weaken the vortex in the inlet pool.

(3) According to the analysis of each section, the bad flow pattern in the forebay led to the secondary deterioration of the flow pattern in the inlet pool and reduced the flow rate in the inlet tank. At this time, the flow velocity is lower than the minimum sediment carrying velocity, which intensifies the continuous settlement of sediment to the bottom of the inlet tank.

(4) According to the comparison between the top view and the longitudinal view of the forebay and inlet pool where sedimentation has formed, if the demand for agricultural irrigation is urgent, then the best course of action is to first start the intermediate pump, and then start the large left pump (with less sediment deposition) to alleviate the water consumption and temporarily improve the operational efficiency of the pump station.

(5) After cross-sectional analysis, a rectifying device is installed in the 1-5 m range after the 
water inlet of the pumping station, which can eliminate the vortex A and vortex B areas to a certain extent, thereby reducing sedimentation and providing good conditions for pumping water.

Through the combination of numerical simulation, theoretical analysis and practical engineering projects, this study analyzes the location and causes of various vortices and puts forward suggestions on the operation of pump stations before and after sediment deposition. This study provides important guidelines that are significant for the improvement and control of flow patterns and sediment deposition in the forebay of a forward intake pump station. In future research, roundabouts and sediment deposition in practical projects in Northwest China can be reduced through further theoretical analysis and experimental measures.

\section{References}

1. Jing-chao, G. U., Liu, X. J., , Li-Guo, L. U., Zhu, J. , \& Hui-Fang, W. U. An analysis of the relationship between water turbidity and sediment in ningxia yellow river. China Rural Water and Hydropower(2016). (In Chinese)

2. Hirsch, \& Charles.. Numerical computation of internal and external flows : Butterworth-Heinemann, 145-201. https://doi.org/10.1016/B978-075066594-0/50045-X(2007).

3. Constantinescu, G. S. , \& Patel, V. C. Numerical model for simulation of pump-intake flow and vortices. Journal of Hydraulic Engineering, 124(2), 123-134. https://doi.org/10.1061/(ASCE)0733-9429(1998)124:2(123) (1998).

4. Hwang, Y. K. , Heo, J. S. , \& Choi, W. J. Numerical and experimental investigations of channel flows in a disk-type drag pump. American Institute of Physics. https://doi.org/10.1063/1.1407655 (2000).

5. Adrian, \& Ronald, J. Hairpin vortex organization in wall turbulence. Physics of Fluids, 19(4), 457. https://doi.org/10.1063/1.2717527 (2007).

6. Xi, W. \& Lu, W. G. Formation mechanism of an adherent vortex in the side pump sump of a pumping station. International Journal of Simulation Modelling, 20(2), 327-338.https://doi.org/10.2507/IJSIMM20-2-562(2021).

7. Jie-min, ZHAN, and, Ben-cheng, WANG, \& and, et al. Numerical investigation of flow patterns in different pump intake systems. Journal of Hydrodynamics Ser B.https://doi.org/10.1016/S1001-6058(11)60315-6(2012).

8. Xue-lin TANG and Wuchang WANG and Fu-jun WANG and Xin YU and Zhi-cong CHEN and Xiao-yan SHI. Application of lbm-sgs model to flows in a pumping-station forebay. Journal of Hydrodynamics, Ser. B. https://doi.org/10.1016/S1001-6058(09)60045-7(2010).

9. Manninen, M. , Taivassalo, V. , Kallio, S. , \& Akademi, A. On the mixture model for multiphase flow vtt publications. (1996).

10. Schiller, L. A drag coefficient correlation. V.D.I. Zeitung, 77. (1935).

11. Kenzakowski, D. , Papp, J. , \& Da Sh, S. Evaluation of advanced turbulence models and variable Prandt//Schmidt number methodology for propulsive flows. Aerospace Sciences Meeting \& Exhibit. (2013).

12. Patankar, S. Numerical heat transfer and fluid flow. Hemisphere Pub. Corp. ; (1980).

13. Rathore, S. K. , \& Das, M. K. Numerical investigation on the performance of low-reynolds number k- model for a buoyancy-opposed wall jet flow. International Journal of Heat \& Mass 
Transfer, 95(5), 636-649. https://doi.org/10.1016/j.ijheatmasstransfer.2015.10.067 (2016).

14. Ansys Inc . Ansys FLUENT theory guide [M] . Southpointe: Ansys Inc (2017).

15. Spalding, B. The numerical computation of turbulent flows. Computer Methods in Applied Mechanics and Engineering. https://doi.org/10.1016/0045-7825(74)90029-2(1974).

16. Yakhot, V. , \& Orszag, S. A. Renormalization group analysis of turbulence. i. basic theory. Journal of Scientific Computing, 1(1), 3-51.https://doi.org/10.1007/BF01061452 (1986).

17. Kim, H. R., Kim, S. , Kim, M. , Park, S. H. , Min, J. K. \& \& Ha, M. Y. . Numerical study of fluid flow and convective heat transfer characteristics in a twisted elliptic tube. Journal of Mechanical Science\&Technology. https://doi.org/10.1007/s12206-016-0127-4(2016).

\section{Acknowledgments}

This work was supported by the National Natural Science Foundation of China (NSFC) under

Grant No. 11761005 and No. 11861003; and the Natural Science Foundation of Ningxia province under Grant No. 2018AAC03043.

\section{Author contributions}

HD.W. analyzed data under the supervision of CG.L. and SJ.L. wrote the paper. LL.S Participated in the measurement data. All authors reviewed the manuscript.

\section{Competing interests}

The authors declare no competing interests.

\section{Data Availability Statement}

All data, models, and codes generated or used during the study appear in the published article. 